\title{
Biophysical Modeling of Information Processing in the Drosophila Olfactory System
}

\author{
Dissertation \\ for the award of the degree \\ "Doctor of phylosophy (Ph.D.) " \\ Division of Mathematics and Natural Sciences \\ of the Georg-August-Universität Göttingen
}

within the doctoral program Theoretical and Computational

Neuroscience

of the Georg-August University School of Science (GAUSS)

submitted by
Faramarz Faghihi

from Tehran (place of birth)

Göttingen, 2013 
Thesis committee

Prof. Dr. Florentin Wörgötter,

Biophysik - III. Physikalisches Institut, Department of Computational Neuroscience, GeorgAugust-Universität Göttingen

Prof. Dr. André Fiala,

Schwann-Schleiden Research Centre, Department of Molecular Neurobiology of Behaviour, Georg-August-Universität Göttingen

Prof. Dr. Martin Göpfert

Schwann-Schleiden Research Centre, Department Cellular Neurobiology of Behaviour, Georg-August-Universität Göttingen

Thesis committee First Reviewer: Prof. Dr. Florentin Wörgötter ,

Second Reviewer: Prof. Dr. André Fiala ,

Other members of the examination board:

\section{Prof. Dr. Ralf Heinrich}

Schwann-Schleiden Research Centre, Department Cellular Neurobiology of Behaviour, Georg-August-Universität ,Göttingen

Prof. Dr. Alexander Gail,

Sensorimotor Group, German Primate Center, Bernstein Center for Computational Neuroscience (BCCN), Göttingen ,

Prof. Dr. Jörg Grosshans , Institute of Biochemistry and Molecular Cell Biology, Department of Developmental Biochemistry, Göttingen University Medical School

\section{Prof. Dr. Halyna Shcherbata}

Max Planck Institute for biophysical Chemistry, Research Group Gene Expression and Signaling, Göttingen

Date of the oral examination: 17.04.2014 


\begin{abstract}
Affidavit
Hereby, I declare that I prepared this doctoral thesis, entitled 'Biophysical Modeling of Information Processing in the Drosophila Olfactory System', on my own and with no other sources than those cited.
\end{abstract}

Faramarz Faghihi (Göttingen, 2013) 
To my wife Sara and my daughter Armita 


\section{Contents}

1 Introduction $\quad \mathbf{9}$

1.1 The structure and function of the Drosophila olfactory system . . . . 10

1.1.1 The antenna and olfactory receptor neurons . . . . . . . . . . 12

1.1.2 The antennal lobe . . . . . . . . . . . . . . . 12

1.1.3 The mushroom body . . . . . . . . . . . . . . . . . 13

1.2 Olfactory Associative Learning . . . . . . . . . . . . . . . . . . 15

2 Aims and related works $\quad 17$

3 An information theoretic model of information processing in the Drosophila $\begin{array}{ll}\text { olfactory system } & 23\end{array}$

3.1 Introduction . . . . . . . . . . . . . . . . . . . 23

3.2 Method ......................... 23

3.2.1 Model structure . . . . . . . . . . . . . . . . . . 23

3.2.2 Odor presentation with different concentration . . . . . . . 25

3.2.3 Feedback inhibition . . . . . . . . . . . . . . 25

3.2.4 Calculation of mutual information . . . . . . . . . . . 26

3.3 Results . . . . . . . . . . . . . . . . . . . . 27

3.3.1 Information transmission under various system parameters without inhibition . . . . . . . . . . . . . . . 27

3.3.2 Information transmission under various system parameters with inhibition . . . . . . . . . . . . . . 28

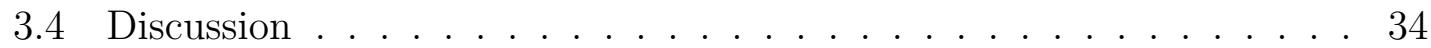

4 A retrograde signaling model of information processing in the Drosophila olfactory learning $\quad 37$

4.1 Introduction . . . . . . . . . . . . . . . . . . 37

4.2 Methods . . . . . . . . . . . . . . . . . . . . . . 40

4.2 .1 Insilico-fly . . . . . . . . . . . . . . . . . . . . . 40

4.2.2 Architecture of the olfactory system . . . . . . . . . . . . 40 
Contents

4.2.3 Neuronal activity models . . . . . . . . . . . . . . . . . . . 41

4.2.4 Retrograde signaling production and diffusion . . . . . . . . . 44

4.2.5 Synaptic efficacy between the antennal lobe and the mushroom body . . . . . . . . . . . . . . . . 45

4.2.6 Dynamics of the synaptic efficacy between the mushroom body and the output neuron. . . . . . . . . . . . . . . 46

4.2 .7 Simulation studies . . . . . . . . . . . . . . 47

4.3 Results . . . . . . . . . . . . . . . . . 52

4.3.1 First phase ....................... 52

4.3 .2 Second phase . . . . . . . . . . . . . . 57

4.3.3 Third phase . . . . . . . . . . . . . . 6 60

4.3.4 Two-dimensional navigation . . . . . . . . . . . . . . 62

4.4 Discussion ............................. 64

5 Conclusion $\quad 67$ 


\section{Abstract}

Fruit flies (Drosophila melanogaster) rely on their olfactory system to process environmental information. Although extensive studies have helped neurobiologists to understand the basic molecular and cellular mechanisms underlying information processing as well as principles of neural circuits of learning in the Drosophila olfactory system, there are still many questions that are awaiting an answer by neuroscience research. Specially, linking the observed behaviors including associative learning to underlying molecular mechanisms and neural circuitry are some challenges of modern neuroscience.

In this research, we have aimed to present some models which are based on available data of the Drosophila olfactory system to describe the role of physiological as well as structural parameters in information procressing in the Drosophila olfactory system. For this purpose, we have presented an information theoretic approach to measure the system efficiency of the Drosophila olfactory system. We have studied the role of some parameters of the this system and of stimuli intensity in the environment with respect to how they influence the transmission of olfactory information. We have designed an abstract model of the antennal lobe, the mushroom body and the feedback inhibitory circuitry. Mutual information between the olfactory environment, simulated in terms of different odor concentrations, and a sub-population of the intrinsic mushroom body neurons (Kenyon cells) was calculated to quantify the efficiency of information transmission. With this method we studied, on the one hand, the effect of different connectivity rates between olfactory projection neurons and firing thresholds of the Kenyon cells. On the other hand, we analyzed the influence of inhibition on mutual information between environment and the mushroom body.

Our simulations show an expected linear relation between the connectivity rate between the antennal lobe and the mushroom body and firing threshold of the Kenyon cells to obtain maximum mutual information for both low and high odor concentrations. However, contradicting our expectations, high odor concentrations cause a drastic, and unrealistic, decrease in mutual information for all connectivity rates compared to low concentration. But when feedback inhibition on the mushroom body is included, mutual information remains at high levels independent of other system parameters. This finding points to a pivotal role of feedback inhibition in Drosophila information processing without which the system efficiency will be substantially reduced.

Associative learning acts as an important information processing mechanism which is known in the Drosophila olfactory system where a given odor is associated with another stimulus as reward or punishment. To model the process in first and secondorder conditioning paradigm, the 'insilico-fly' is introduced. These flies are neural networks that have been constructed using the available knowledge about neuronal 


\section{Contents}

systems capable to produce both, first and second-order, associative learning. This model is based on the integration of synaptic and non-synaptic neural communications. The non-synaptic neural communication includes retrograde signal that may change the synaptic weight at both candidate places for associative learning in the Drosophila brain : 1 . the synapses between the Kenyon cells and the output neuron and 2. the synaptic weights between the Kenyon cells and the projection neurons). In this model, the increased synaptic weight in both learning places are necessary to produce second-order conditioning, but after training, removing the synaptic weight between the Kenyon cells and the projection neurons does not impair second-order conditioning because these synapses gets high weight after presenting the trained odor to the neural system. The insilico-fly takes advantage of this feature because it allows specific association between odors and punishment (different odors may share some projection neurons). The insilico-fly illustrates the importance of integrating of systems biology and computational neuroscience to investigate complex mechanisms of behavioral studies in both Drosophila olfactory system or higher animals. 


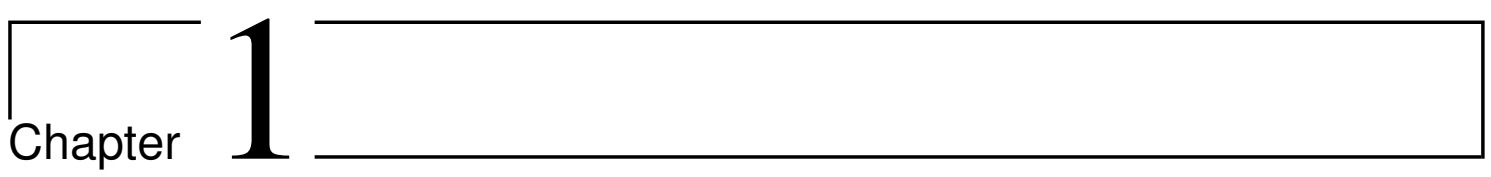

\section{Introduction}

The animals' brain constructs the representation of environmental stimuli by complicated information processing mechanisms of their neural systems. Sensory systems map features of the living environments into neural representation in animals' brain. This process which is called 'neural encoding' allows animals to navigate their dynamical environments to search for their needs. Neuroscience researchers have aimed to understand how sensory information is transformed from initial detection, through various stages of neural information processing to generation of a percept that drives specific behavior.

Olfaction can be an evocative sense for humans, but for many animals species, including insects, it is a vital sense for reproduction, feeding and avoiding dangers. The chemical senses- taste and olfaction- comparing to other senses, use a different strategy to represent information because categorize both the quality and intensity of the stimulus [Benton, 2008], [Keene and Waddell, 2007]. Animal's olfactory systems have been evolved to detect weak and fluctuating signals (large number of volatile chemicals) in natural environments [Firestein, 2001]. An important feature of the olfactory systems that differentiates them from other sensory systems of animals' brain is that 'time' can be used as a coding dimension for the representation of non-temporal feature of odors [Laurent, 1999].

There some reasons why the study of olfactory system is crucial for neuroscience researches to understand the principles of information processing in animals' brain [Kivity et al., 2009]. The first reason is the very striking homology in the structure and function of the olfactory systems between different animal species, including insects and mammals. These observations have motivated neuroscientists to consider the possibility of existing common mechanisms for olfactory perception, discrimination, and associative learning in the animals' olfactory systems. The second advantage for studying the olfactory systems is that many animals exhibit the ability to form olfactory memories and associative learning [Brennan and Keverne, 1997], 


\section{Introduction}

[Laurent et al., 2001a], [Mombaerts, 2001].

The manageable size of the Drosophila Melanogaster (briefly Drosophila) compared to vertebrates' brain, the comprehensive description of the Drosophila olfactory system (including molecular description of olfactory receptor neurons), available molecular genetic techniques and recording neural activity technology have made it a fascinating animal model to study the principles of information processing in neural systems [Olsen and Wilson, 2008]. Thus, studies of the Drosophila olfactory system can shed light on fundamental principles of sensory information processing in all brains [Olsen and Wilson, 2008], [Singer et al., 2009],[Kay and Stopfer, 2006].

Furthermore, the Drosophila olfactory system has been studied by computational neuroscientists to develop models and theories about how olfactory information is processed and transformed to elicit a specific behavior. The common focus of all studies on the Drosophila olfactory system is the mechanisms of neural encoding within brain and how different odors (with different concentrations) are represented in the system in terms of neuronal activity and plasticity of neural connections.

We first summarize the structure and function of different parts of the Drosophila olfactory system then the studies of the information processing and associative learning in Drosophila olfactory system are discussed.

\subsection{The structure and function of the Drosophila olfactory system}

A description of information processing in the neural systems depends on understanding the relevant circuits' layout, so we should first consider the knowledge about the anatomy and physiology of the Drosophila olfactory system. Intensive studies have shown the structure and function of many parts of this neural system [Mcguire et al., 2005]. A schematic for basic anatomy of the Drosophila olfactory system and their neural architecture which includes 'the antenna', 'the antennal lobe', 'the mushroom mody', 'the lateral horn' and 'the output neurons' is shown in Fig. 1.1. Schematically, the mushroom body's input can be described as a scaffold, with arrays of projection neuron axons crossing arrays of Kenyon cells, and forming synapses with some Kenyon cells [Heisenberg, 2003]. In the following sections, a brief description of the physiology of these parts of the Drosophila olfactory system is presented. 


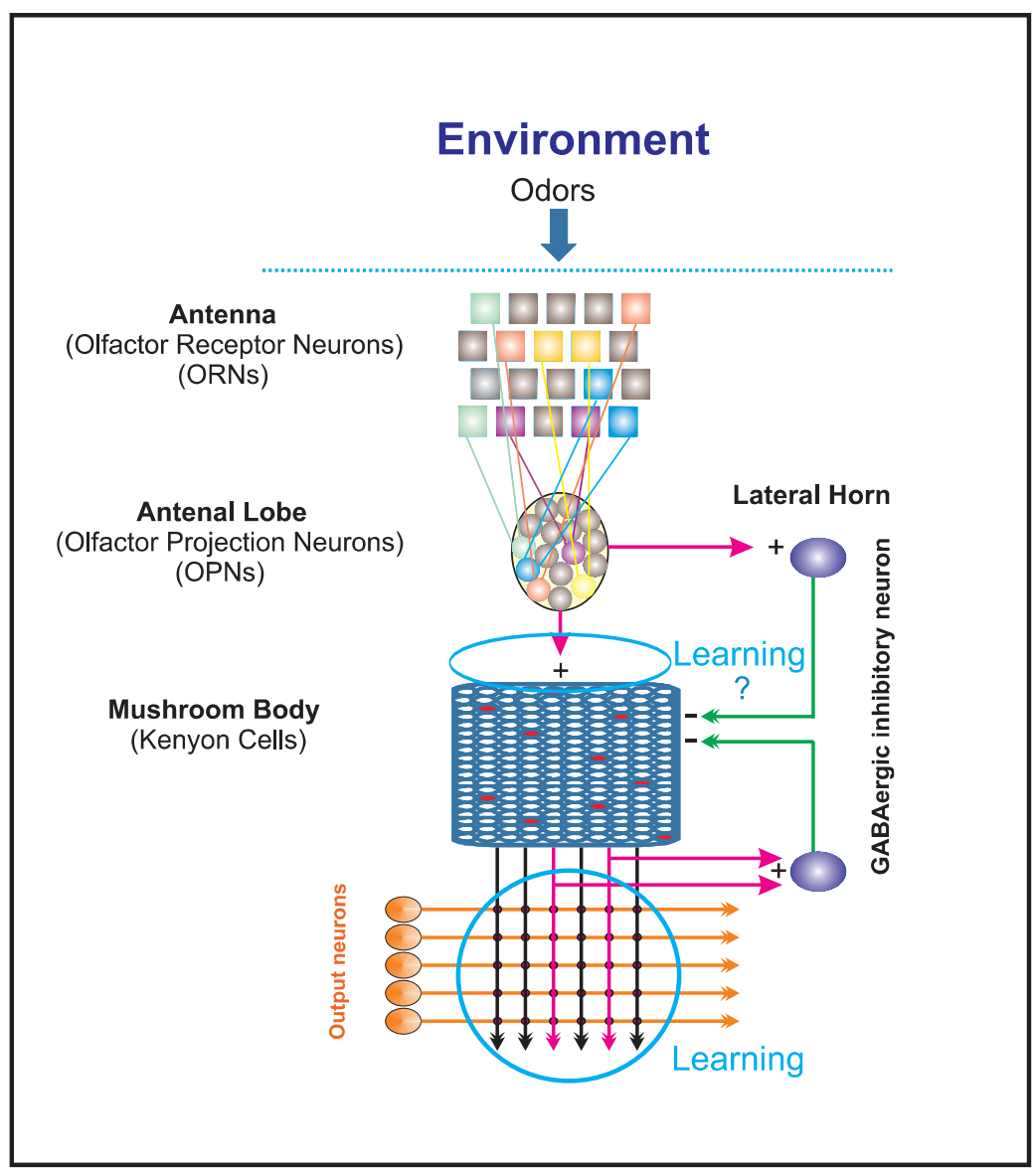

Figure 1.1: Schematic illustration of the Drosophila olfactory system. Odor as a stimulus in the environment binds to the receptors located on the antenna; each olfactory receptor neuron expresses one specific type of receptor (illustrated by different colors). Olfactory receptor neurons of the same class project to one glomeruli in the antennal lobe. The projection neurons in the antennal lobe, in turn, activate the Kenyon cells in the mushroom body and the lateral horn by cholinergic, excitatory synapses. GABAergic inhibitory neurons provide inhibitory effects on the activated Kenyon cells. The synapses between the output neuron and the Kenyon cells is the area where learning takes place. Another candidate for the learning place is the synapses between the Kenyon cells and the projection neurons. 


\section{Introduction}

\subsubsection{The antenna and olfactory receptor neurons}

The binding of volatile molecules (odors) in environment to the receptor proteins on the surface of the dendrites of neurons in the antenna [Vosshall et al., 2000], [Vosshall et al., 1999] which are called Olfactory Receptor Neurons (ORNs) leads to generation of spikes [Benton et al., 2009] which are subsequently sent to the inner parts of the insects' brain. All of the information about the quality and concentration of an odor is represented in the population activity of ORNs. The neurotransmitter used by ORNs is believed to be acetylcholine, which is the primary excitatory transmitter in the insects' brain [Bicker, 1999]. Although some ORNs respond to specific chemicals, other ORNs respond to a wide range of odors that do not share a set of common features [Hallem and Carlson, 2006], [Malnic et al., 1999],[Yao et al., 2005]. Drosophila has two pairs of olfactory organs, the antenna and the maxillary palps. Each antenna contains about 1200 ORNs while the maxillary palp has about 120 ORNs. In Drosophila one receptor gene, Or83b is expressed in most ORNs where it is required for their detection capability. The large odorant receptor family is not expressed at random in individual ORNs; rather, each ORN typically expresses normally one type of receptor protein and converge to a single glomerulus in the antennal lobe [Couto et al., 2005], [Fishilevich and Vosshall, 2005].

This feature has been used to develop models about the convergence and the divergence of neuronal information in the Drosophila olfactory system

[García-Sanchez and Huerta, 2003].

\subsubsection{The antennal lobe}

The antennal lobe is a common structure in the olfactory system of all insects including Drosophila which is involved in olfactory information processing [Strausfeld and Hildebrand, 1999]. The antennal lobe is analogous in structure and function to the vertebrate olfactory bulb [Strausfeld and Hildebrand, 1999]. While the mammalian bulb is structured in layers, the insects' antennal lobe is structured entirely in glomerular units which acts as the interaction site of ORN, the local neurons and the projection neurons. The total number of the projection neurons in Drosophila is estimated to be between 150 to 200 [Stocker et al., 1997]. The antennal lobe performs some important functions: 1) the input noise can be reduced by signal averaging [Laurent, 1999], 2) using widespread local inhibition, the antennal lobe circuits can compress the dynamic range of its projection neurons output over many orders of magnitude of input [Stopfer et al., 2003]. These operations are important as first steps in the processing of odor signals. However, because the projection neurons are broadly tuned, odor encoding at the level of the antennal lobe's output is densely combinatorial and thus inefficient for storage. The projection neurons are spontaneously active in Drosophila [Wilson et al., 2004] and their responses are 
shaped by inhibitory network within the antennal lobe [Wilson and Laurent, 2005] , [Silbering et al., 2008], [Schlief and Wilson, 2007],[Bhandawat et al., 2007].

The projection neurons are the only neurons that send odor's information to the mushroom body and the lateral horn. In Drosophila, the projection neurons' dendrites usually innervate single glomerulus [Stocker et al., 1990]. Therefore, they receive the input from the olfactory receptor neurons that express the same olfactory receptor. In Drosophila, each glomerulus receives bilateral input from an average of 50 ORNs (25 per antenna) expressing same olfactory receptor where they synapse with an average of three projection neurons. It is believed that each ORN contacts all the projection neurons in a glomerulus [Vosshall, 2000]. The local neurons can be either inhibitory or excitatory, releasing GABA or acetylcholine, respectively [Shang et al., 2007],[Olsen et al., 2007]. The local neurons are about 100 GABAergic neurons in Drosophila [Ng et al., 2002]. It has been found that the local neurons receive input from both ORNs and the projection neurons [Wilson and Laurent, 2005]. Another noticeable feature of the antennal lobe is that number, shape and arrangement of glomeruli is a strongly specific property: adult Drosophila has about 50 glomeruli [Laissue et al., 1999].

Another chemical that is produced in insects' brain is nitric oxide. The roles of this gaseous chemical in insects' brain are not clearly known [Bicker, 2001],

[Gage et al., 2013]. The nitric Oxide has been found in insect's antennal lobes. To investigate the role of nitric oxide in olfactory information processing, nitric oxide production was blocked which disrupted olfactory discrimination [Bicker, 2001].

\subsubsection{The mushroom body}

As mentioned, in Drosophila the olfactory sensory neurons project to the antennal lobe; olfactory sensory neuron of a given type converge in regions called glomeruli. In glomeruli they contact the projection neurons and the local interneurons. The local interneurons which are inhibitory [Python and Stocker, 2002],

[Wilson and Laurent, 2005] or excitatory [Shang et al., 2007] form widespread connections within the antennal lobe whereas projection neurons transfer olfactory information to deeper circuits in the brain, including the Kenyon cells of the mushroom body [Marin et al., 2002], [Wong et al., 2002].

In Drosophila there are about 2500 Kenyon cells [Stocker, 1994]. The Kenyon cell morphology, pharmacology and peptide expression show a considerable variability across all species studied so far [Sinakevitch et al., 2001], [Strausfeld et al., 2003]. The invariant circuitry of the lateral horn is thought to mediate innate behaviours [Jefferis et al., 2007], whereas the mushroom body translates olfactory sensory information into learned behavioural responses. The projection neurons' axons that innervate the mushroom body terminate in large boutons [Wong et al., 2002], 


\section{Introduction}

[Marin et al., 2002] that synapse on the Kenyon cells [Butcher et al., 2012], [Leiss et al., 2009],[Yusuyama and Meinertzhagen, 2002].

The Kenyon cells can be subdivided in different sub-classes: those projecting directly to $\gamma$ and those bifurcate in $\alpha / \beta, \alpha^{\prime} / \beta^{\prime}$ where it synapses onto a relatively small number of the extrinsic output neuron [Tanaka et al., 2008], [Séjourné et al., 2011]. The mushroom Body receives both olfactory and visual input in most insect species and so may play critical roles in other kinds of learning tasks [Farris, 2005],

[Strausfeld and Hildebrand, 1999]. It is well known that the mushroom body is also involved in associative learning [Busto et al., 2010],[Heisenberg, 2003],[Fiala, 2007]. Insects that lack normally developed mushroom body suffer from learning and memory deficits [Heisenberg et al., 1985].

Electrophysiological and optical imaging studies show that olfactory sensory system creates representation of olfactory stimulus as activities of a sub-population of the Kenyon cells such that each activated Kenyon cell generates a few spikes [Jortner et al., 2007], [Stopfer et al., 2003], [Turner et al., 2008].

While the projection neurons had a response probability of 0.64 , the Kenyon cells responded with probability of 0.11 to a given odor set [Perez-Orive et al., 2002], [Szyszka et al., 2005], [Wang et al., 2004]. The baseline activity of Kenyon cells is close to zero [Perez-Orive et al., 2002], [Broome et al., 2006] and their response to odors contain on average 5 spikes, moreover, their output synapses of Kenyon cells in both high or average are modifiable by Hebbian learning rule

[Cassenaer and Laurent, 2007].

In addition, odor representation is dense in the antennal lobe but sparse in the mushroom body. This phenomenon is called 'sparse coding' provides numerous computational advantages for sensory representation and memory storage

[Perez-Orive et al., 2002]. In other word, sparsely encoding by the Kenyon cells exhibits a high level of odor selectivity [Wang et al., 2004],[Honegger et al., 2011]. Regarding of these observations, the question of interests is: How sparse coding arises from the fundamental structural and physiological features of the neural system?

Synaptic connection from the antenna lobe to the Kenyon cells dendrites may be the sites of functional plasticity relevant to the acquisition of memories. Synaptic plasticity on the Kenyon cells dendrites is important to ensure optimal sparse responses in the mushroom body after sufficient odor presentations [Finelli et al., 2008]. Presynaptic mechanism of plasticity between the Kenyon cells and the mushroom body extrinsic neurons as a critical process in olfactory memory is well known [McGuire et al., 2003], [Menzel and Manz, 2005], [Zars, 2000].

Thus, the large number of Kenyon cells allows for the computation of highly nonlinear classification schemes across projection neurons [Huerta et al., 2004]. Temporal complexity can increase the theoretical capacity of the system even further 
[Laurent et al., 2001b]. This organization of glomerular connections to the mushroom body could allow the fly to contextualize novel sensory experiences, a feature consistent with the role of this brain center in mediating learned olfactory associations and behaviors. There is a normalization negative feedback loop within the mushroom body of Locust to maintain sparse output over a wide range of input conditioning [Papadopoulou et al., 2011]. It is known that GABAergic system plays important role in information processing in the mushroom body [Ren et al., 2012], [Liu et al., 2007]. GABAergic system acts as the major inhibitory neurotransmitter in the central nervous system of Drosophila and was expressed in a pair of Anterior Paired Lateral (APL) neurons that innervated the entire mushroom body [Lee et al., 2003],

[Liu et al., 2009], [Lin et al., 2014]. The molecular mechanism of GABAergic system to modulates sparse coding in the mushroom body is not clearly understood. APL neurons are believed to provide direct feedback inhibition to the Kenyon cells [Guo et al., 1996],[Stocker et al., 1997].

\subsection{Olfactory Associative Learning}

Learning can be defined as a lasting alteration in behavior or in the behavioral potential due to experience. An understanding of associative conditioning is a fundamental research in neuroscience. By using an extensive set of experimental approaches in different model organisms, neuroscientists have tried to understand how behavioral changes are represented in terms of molecular and neuronal architecture in the brain [Milner et al., 1998],[Heisenberg, 2003], [Keene and Waddell, 2007].

Drosophila exhibit the conditioned approach or avoidance to an odor that has been paired to an appetitive or aversive stimulus, such as sugar [Tempel et al., 1983] or shock [Tully and Quinn, 1985]. The mushroom body have many tasks beyond olfaction: they are used for spatial memory and navigation without olfactory cues [Kwon, 2004]. Learning deficits have been observed in the Drosophila mutants where the mushroom body structure is altered [Heisenberg et al., 1985]. Blocking synaptic activity or disrupting the mushroom body physiology also leads to memory deficits [Dubnau et al., 2001].

Inhibitory mushroom body output/feedback neuron are necessary for memory formation, and when they are silenced, no memory is formed [Liu and Davis, 2009]. The inactivating of the mushroom body by blocking synaptic transmission from the Kenyon cells prevents insects from forming associative learning

[Heisenberg, 2003], [Davis, 2005],[Krashes et al., 2007].

It is important to notice that aversive conditioning leads to changes in the antennal lobe, where the projection neurons in some glomeruli change their odor responses for about three minute after conditioning [Yu et al., 2004].

The most commonly used associative learning paradigm in Drosophila relies on a 


\section{Introduction}

differential Pavlovian conditioning procedure [Tully and Quinn, 1985] in which an odor (Conditioned Stimulus+ or CS+) is temporally paired with electric shocks (Unconditioned Stimulus or US). A second odor is presented equally often, but without any punishment (Conditioned Stimulus- or CS-). In a subsequent choice test, flies avoid the odor associated with the punishment. The only factor that distinguishes $\mathrm{CS}+$ from CS- is the coincident occurrence of the punishment. Consequently, the change in behavior can be attributed to the coincidence of CS+ and US in contrast to CS alone. As shown by numerous reports, Drosophila is able to establish simple forms of appetitive and aversive olfactory associations at both larval and adult stages [Pauls et al., 2010],[Aceves-Piña and Quinn, 1979],[Gerber and Stocker, 2007],

[Khurana et al., 2009],[Selcho et al., 2009].

Olfactory associative conditioning in Drosophila larvae is well-established for the study of Pavlovian conditioning

[Aceves-Piña and Quinn, 1979],[Gerber and Stocker, 2007],

[Khurana et al., 2009], [Selcho et al., 2009].

Associative conditioning in Drosophila melanogaster has been well documented for several decades. However, most studies report only simple associations between the conditioned stimuli (CS, e.g., odor) and the unconditioned stimuli (US, e.g., electric shock) to measure learning or establish memory. The studies on second order conditioning present an opportunity for reassessing the roles of known genes involved in learning and memory as well as neuronal circuitry involved in a behavioral paradigm. The ability to form higher-order associations is prevalent in nature. second-order conditioning studies have spanned numerous animal models, including honeybees [Abramson et al., 2010], [Hussaini et al., 2007a] ,crickets [Mizunami et al., 2009], pigeons [Rahotte et al., 1977], rats [Debiec et al., 2006]

and humans [Jara et al., 2006],[Karazinov and Boakes, 2007].

Although second-order conditioning was originally described by Pavlov and has been thoroughly studied by psychologists for nearly four decades modern neuroscience has only recently turned to this paradigm as a means for understanding the mechanisms of learning and memory [Gewirtz, 2000].

Second-order conditioning is a form of higher-order associative learning wherein a previously conditioned stimulus (CS1) can associate with a second conditioned stimulus (CS2) to elicit a conditioned response. Initial training involves pairing of CS1 with an unconditioned stimulus (US) during a first-order conditioning session followed by a second-order conditioning session in which CS1 is paired subsequently with a novel stimulus, CS2. If second-order conditioning is successful, an animal will demonstrate a conditioned response to CS2 similar to CS1, even though it has not been exposed to the original US during CS2 and CS1 association [Tabone and Belle, 2011]. 


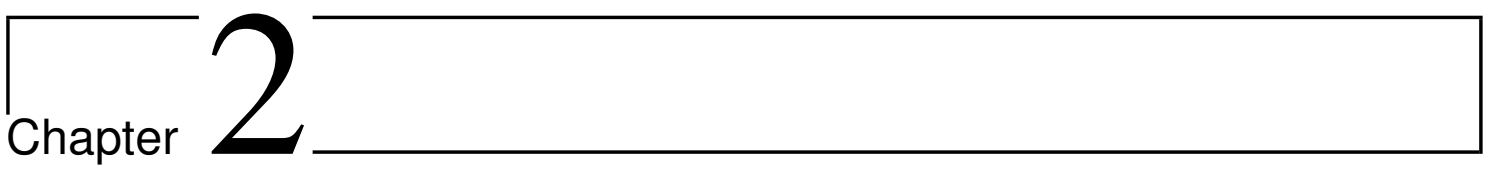

\section{Aims and related works}

Modern neuroscience has aimed to understand how animals' brain process information from the environment to generate an appropriate behavior to adapt themselves to live in a dynamic environment. For this purpose, the Drosophila brain has been studied by neuroscience researchers as a model to study the principle of information processing in neural systems, due to its low number of neurons compared to higher animals and the available experimental techniques. Although many facts about the structure and physiology of the Drosophila olfactory system are already known, there are still some questions awaiting an answer:

1. How is the 'sensory information' received by sensory receptor cells and transferred to higher brain regions to elicit appropriate reactions?

2. How can the system efficiency of the Drosophila olfactory system to transfer odors' information into learning centers be measured to study the role of different structural and physiological parameters. Specially, to predict the optimal value of each parameter is highly of interest.

3. What are the cellular and network mechanisms underlying complex capabilities of Drosophila. For example, understanding of second-order conditioning in the Drosophila olfactory system is a fascinating information processing mechanism that is believed to shed light on neural mechanisms underlying complicated behaviors in animals' brain.

Experimental techniques have limits to test different hypothesis about the occurred neural mechanisms. For example, neural network connectivity may have important role in sensory processing [Wall and Glackin, 2013]. But it is impossible to change it in a given animal to study its role in information processing. Another example is the firing threshold of neurons, which as a biophysical property depends on the expression of different genes in neurons. Neural spiking models help to simulate a neuron's activity and provides the possibilities to change some of structural and 


\section{Aims and related works}

physiological parameters. Modifying these structural and physiological parameters is required to find optimal system efficiency of the neural system to do different learning tasks. The structural parameters include the number of neurons in each part of the neural system and the connectivity rate of neural layers. The physiological parameters include spiking activity of neurons, threshold of firing and inhibitory activity of feedback or feedforward neurons.

The simulation of the neural networks in the brain is necessary to develop hypotheses and to test them for associative learning in the Drosophila olfactory system. In the simulations, the knowledge of the structure and function of different parts is used while some unknown parameters or neural mechanisms are modeled to study their role in information processing.

Theoretical studies have been done to study the structure and function of the Drosophila olfactory system: [García-Sanchez and Huerta, 2003] have studied the parameters of the sensory system to determine information and energy costs and to determine the structural parameters that produce specific responses as well as to find the optimal threshold of the Kenyon cells. [Gu and Liljenström, 2007] have constructed dynamical neural networks to simulate presentation, amplification and discrimination of odors.

[Huerta and Nowotny, 2009],[Huerta et al., 2004] constructed models for pattern recognition in the insect brain to investigate which features are useful to learn the input data and structural organization suitable for fast learning. [Huerta et al., 2004] have shown the range of activity and size of the network required to achieve efficient odor classification in the insect brain. Biological control mechanism can accomplish efficient classification of odors. [Brody and Hopfield, 2003] presented an algorithm for robust odor recognition where main features of their model are taken from known properties of biologically olfactory systems. Information theory has been used in modeling of information preservation in the antennal lobe in the Drosophila olfactory system too [Satoh et al., 2010].

In a similar attempt, we have aimed to develop an information theoretic model to measure the system efficiency using experimental data of molecular, cellular, and network mechanisms in the Drosophila olfactory system. The system's parameters that are highly interesting to understand with respect to their role in the animals life are connectivity rate between the antennal lobe and the mushroom body, threshold of firing of neurons and the inhibitory neurons. To find the optimal values for the parameters that correspond to maximum system efficiency is of central relevance. Specially, we are interested in investigating the role of feedback inhibition in the system efficiency to encode different odor concentration (Fig. 2.1).

Associative learning is an important information processing mechanism. A model for second-order conditioning in crickets has been proposed which considers two conditioned stimuli; olfactory and visual and pairs them with reward or punishment 
which are presented by the neural activity of octopaminergic and dopaminergic neurons, respectively. In the proposed model two kinds of synaptic connections are formed during conditioning:

1) the connection from neurons that represent conditioned stimulus to dopaminergic neurons which is called 'stimulus-stimulus connection' and 2) the connections from neurons representing the conditioned stimulus to neurons that induce the conditioned response (avoidance behavior)(Fig. 2.2). This model and the experimental support have suggested a similar mechanism of conditioning in insects and higher vertebrates. Briefly, the model assumes the formation of connections between the first conditioned stimulus and dopaminergic neurons in the first stage of conditioning, which allows the first conditioned stimulus to activate dopaminergic neurons and so substitute for the punishment in the second stage of the conditioning (pairing of first and second conditioned stimuli) [Mizunami et al., 2009]. Experiments are still requested to test this model in second-order conditioning in the Drosophila olfactory system [Tabone and Belle, 2011].

To model associative learning in the Drosophila olfactory system, a retrograde signaling based model has been proposed that assumes change of intrinsic plasticity of the Kenyon cells induced by diffusion of the retrograde signal from the output neuron to the Kenyon cells [Smith et al., 2008]. We have developed a retrograde signaling based model for second-order conditioning in which synaptic plasticity at both learning sites in the Drosophila olfactory system occur (Fig. 2.3). This model, on the one hand, offers some hypothetical mechanisms of learning and information processing that should be tested by experimental techniques, and on the other hand, it presents some learning rules to implement insects' capabilities in artificial systems. In next chapters, these models are described and discussed in details. 
2 Aims and related works

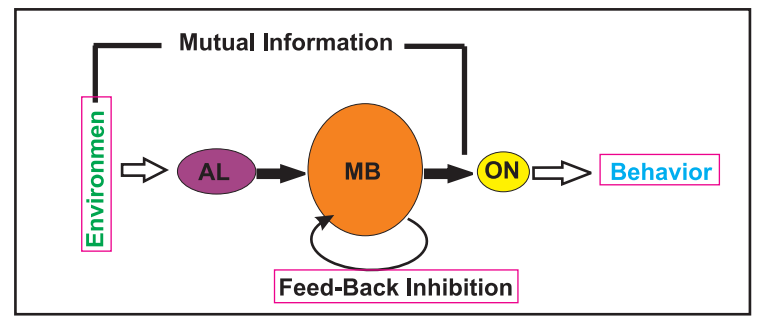

Figure 2.1: Information theoretic model of the Drosophila olfactory system. The connectivity rate between the antennal lobe (AL) and the mushroom body (MB), threshold of firing of the Kenyon cells and feedback inhibition are the parameters of the model. Mutual information between environment and the mushroom body is measured. 

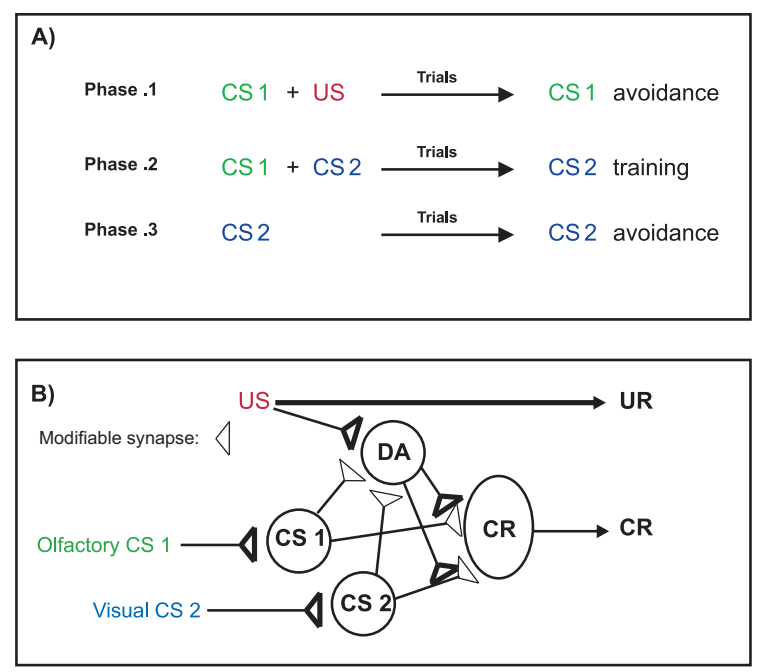

Figure 2.2: A) The second-order conditioning behavioral paradigm. First an odor is paired with an electric shock for few trials. Then the first and the second odor (null stimulus) is presented to the fly in the absence of the electric shock. After some trials, the animal learns to avoid the second odor. B) Mizunami-Unoki model for second-order conditioning. This model assumes the formation of connections between first conditioned stimulus and dopaminergic neurons in the first stage of conditioning, which allows the first conditioned stimulus to activate dopaminergic neurons and so substitute for the punishment in the second stage of conditioning (pairing of first and second conditioned stimulus).

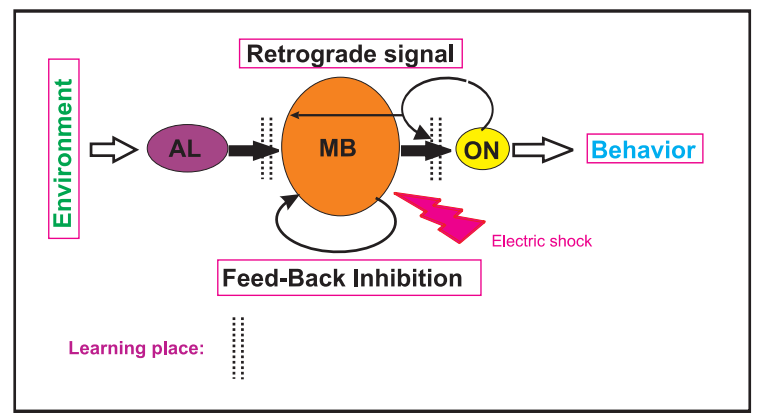

Figure 2.3: The structure of the proposed model for second-order conditioning. The model is basically the same as that for first order conditioning but it is assumed that the retrograde signal from the output neuron into the Kenyon cells trigger some biochemical reactions that leads to the change in the synaptic weight between the antennal lobe and the mushroom body. 

Chapter 2

\section{An information theoretic model of information processing in the Drosophila olfactory system}

\subsection{Introduction}

In this work, we analyze how much environmental information the Drosophila olfactory system transmits to the mushroom body and, hence, to the output neurons. Several physiological parameters influence this transmission in different ways. Here, we determine the influence of the connectivity rate between OPNs and Kenyon cells and the firing threshold of the Kenyon cells. Specifically, the impact of different strengths of inhibiting Kenyon cells firing is assessed. To quantify the transmission efficiency, we calculated the mutual information for the different conditions between the environmental input and the mushroom body output.

In the following, we present the structure of the model, the implementation of inhibition, and the way of calculation mutual information.

\subsection{Method}

\subsubsection{Model structure}

The simplified anatomical structure used in this model is presented in (Fig. 3.1). We combined the antenna and the antennal lobe in a circuit because the ORNs, which express the same specific olfactory receptors, send their axons to the same 
glomeruli, and OPNs in each glomerulus receive information just from ORNs of the same class. Reported exceptions from these rules, i.e., ORNs expressing more than one receptor, ORNs targeting more than one glomerulus and multiglomerular OPNs receiving information from many glomeruli, are disregarded for simplicity. Furthermore, we assume that each of the 50 glomeruli [Caron et al., 2013] consists of one OPN.

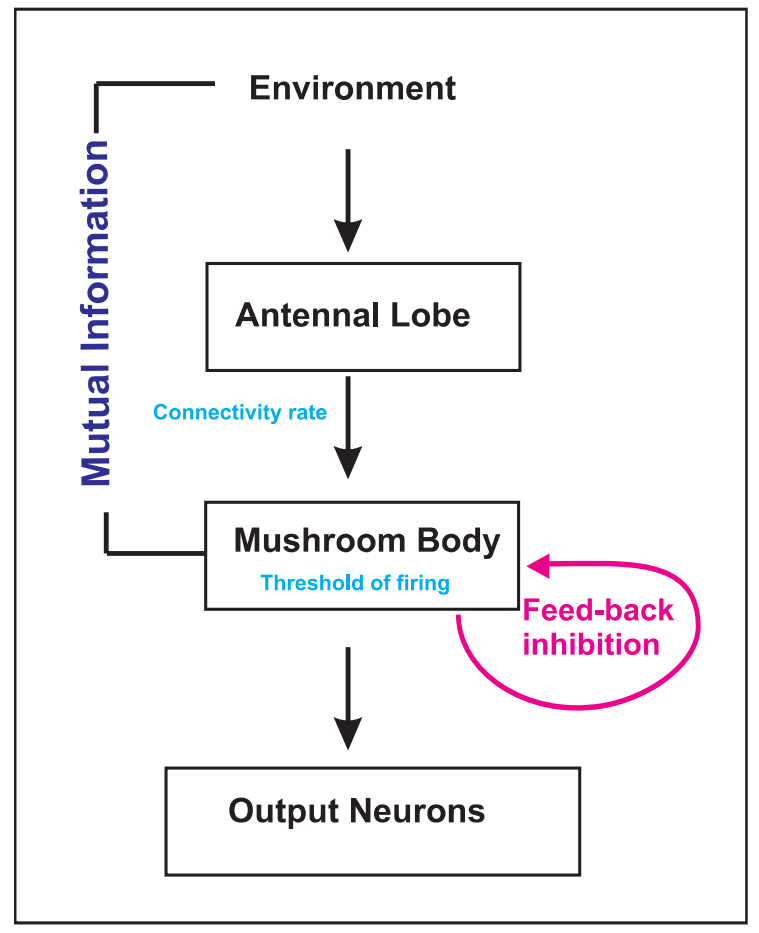

Figure 3.1: Schematic illustration of the model structure in information theoretic approach. Odor information in the environment is sent via the antenna to the antennal lobe and subsequently to the mushroom body. Inhibitory neurons contribute to the information processing. There are two known inhibitory connections to the mushroom body calyx originating either from the lateral horn or from the mushroom body itself. Mutual Information between the environment and Kenyon cells is measured for different activation threshold, connectivity rates, and inhibition strengths.

Thus, in our model, an odor $k$ presented to the circuit reaches a subset of glomeruli or OPNs as odors consists of several different chemicals. These diverse odor structures yield different numbers of reached OPNs $N_{O P N}^{k}$. These OPNs are the subgroup which can be activated by the odor $k$. Their number is drawn from a Gaussian distribution with mean $\mu_{O P N}=35$ and variance $\sigma_{O P N}^{2}=8$. This group of OPNs, in turn, projects to a subgroup of Kenyon cells in the mushroom body. As we show below, the exact number of Kenyon cells $N_{K C}$ does not alter the results qualitatively. 


\subsubsection{Odor presentation with different concentration}

As described before, each odor $k$ presented to the system activates a subgroup of neurons in the antennal lobe. Each OPN $j$ can be either active $\chi_{j}^{k}=1$ or silent $\chi_{j}^{k}=0$. The number of activated OPNs depends on the concentration $c_{\text {odor }}^{k}$ of the odor. Low concentrations $\left(c_{\text {odor }}^{k} \approx 0\right)$ activate a few, high concentrations $\left(c_{\text {odor }}^{k} \approx 1\right)$ activate nearly all reached OPNs. However, due to noise, this number can vary over trials. Thus, the activation of each reached OPN is probabilistic dependent on the following equation:

$$
p_{O P N}^{k}=1-e^{-\beta \cdot c_{o d o r}^{k}} .
$$

The parameter $\beta$ determines the relation between odor concentration and neuronal activity and, without loss of generality, is set equal to 1.32. Thus, each odor is represented in the antennal lobe as a state of $1 \mathrm{~s}$ and $0 \mathrm{~s}$ of length $N_{O P N}^{k}$. This state is transmitted via the synapses in the calyx to the Kenyon cells. Thereby, each OPN $j$ connects to Kenyon cell $i$ with probability $r$ (drawn from the interval from zero to one without boundaries) which defines the connectivity rate. Thus, the connection $c_{i, j}$ is either zero or one depending on $r$. All $N_{K C}$ Kenyon cells are modeled as simple integrate-and-fire neurons with firing threshold $\Theta$ (between 1 and 20):

$$
\psi_{i}^{k}= \begin{cases}1 & \text { if } \sum_{j}^{N_{O P N}^{k}} c_{i, j} \cdot \chi_{j}^{k}>\Theta \\ 0 & \text { if } \sum_{j}^{N_{O P N}^{k}} c_{i, j} \cdot \chi_{j}^{k} \leq \Theta .\end{cases}
$$

Thus, each odor yields via the antenna and antennal lobe to a specific firing pattern of Kenyon cells in the mushroom body.

\subsubsection{Feedback inhibition}

To assess the influence of different inhibitory strengths on mushroom body, we introduce a probability $p_{I}^{k}$ that Kenyon cells firing can be inhibited. This probability depends on the average activity in the mushroom body $\tilde{\chi}^{k}$ without inhibition.

$$
p_{I}^{k}=e^{-\frac{\alpha}{\tilde{\chi}^{k}}}
$$

The parameter $\alpha$ (between 0.05 and 1.75) determines the different strengths of inhibition (Fig. 3.2). Thus, we first derive the activity of the Kenyon cells as described before, calculate the probability of inhibiting each Kenyon cell spike, and derive the new Kenyon cell firing. This new state, dependent on the inhibitory feedback, is the final output of the mushroom body. 


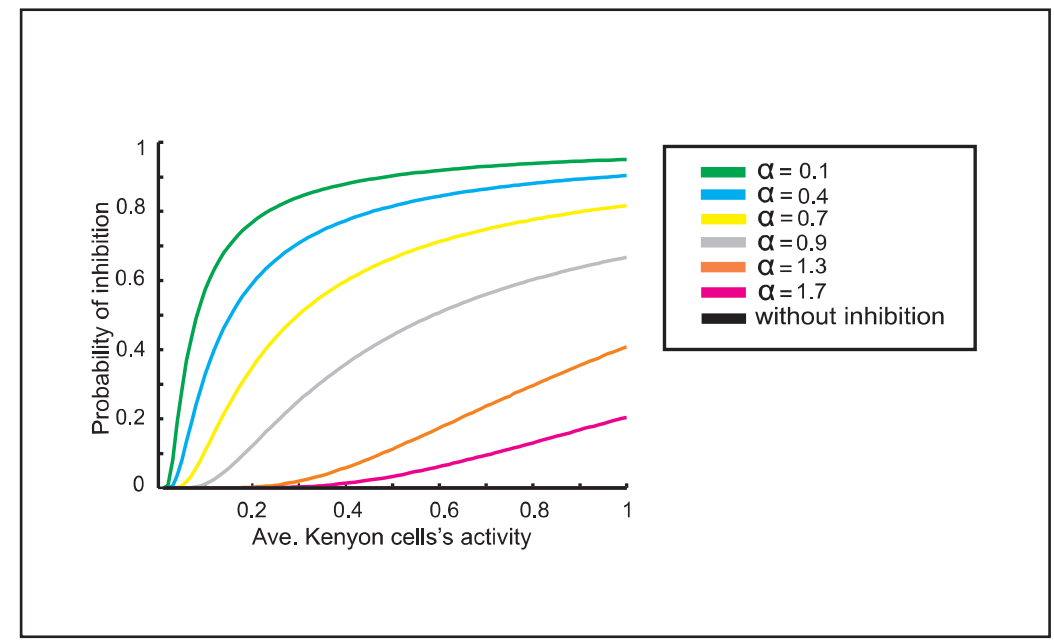

Figure 3.2: The relation between average Kenyon cells activity and probability of feedback inhibition for different parameter values $\alpha$. Note, a higher value of $\alpha$ induces less inhibition.

\subsubsection{Calculation of mutual information}

From information theoretic point of view the Drosophila melongaster olfactory system can be considered as an 'information channel' which transmits environmental information to the mushroom body. The efficiency of such a channel can be characterized by 'mutual information'. To calculate mutual information between the environment (odors) and the mushroom body (Kenyon cells) we need to assess the probability distribution of Kenyon cells firing $p(n)$. Each $n$ represents one out of $\mathfrak{N}=2^{N_{K C}}$ states of neuronal activity in the mushroom body. The distribution of Kenyon cells firing depends on the information from the environment. This information is represented by the probability distribution $p(k)$ of $\mathfrak{K}$ odors presented to the olfactory system. In the following, we assume that these odors are equiprobable. Thus, the mutual information between the environment and the mushroom body is

$$
M I=\sum_{n=1}^{\mathfrak{N}} \sum_{k=1}^{\mathfrak{K}} p(n, k) \log \left(\frac{p(n, k)}{p(n) p(k)}\right) .
$$

$p(n, k)$ is the joint probability distribution of Kenyon cells states and presented odors. Note, to guarantee calculability we set $p(n)=10^{-8}$ when state $n$ does not occur.

In this study, we tested the efficiency of information transmission dependent on several physiological and environmental parameters as average connectivity $r$ between antennal lobe and mushroom body, firing threshold $\Theta$ of Kenyon cells, inhibition (parameter $\alpha$ ), number of Kenyon cells $N_{K C}$, and odor concentration $c_{o d o r}$. As the actual connectivity is created probabilistically (see before), we tested 20 different 
systems ('flies') with each having another connectivity for one set of $\Theta, r, \alpha, c_{\text {odor }}$, and $N_{K C}$. Each system was tested in 100 trials with 100 different odors of same concentration. The odors are specified by the number of OPNs reached. In every trial a different subset of the reached OPNs becomes active.

Since the 100 trials does not provide sufficient statistics to assess the joint probability distribution $p(n, k)$ (how often which state $n$ occurs given odor $k$ ) and the probability distribution of Kenyon cells firing $p(n)$ (how often each state $n$ occurs in total) we used the 'Quadratic Extrapolation procedure' to correct for the sampling bias problem [Panzeri et al., 2007]. This procedure assumes that the biased mutual information $M I_{\text {uncorrected }}$ can be approximated as expansion in $\frac{1}{N}$, where $N$ is the number of trials. That is

$$
M I_{\text {corrected }}=M I_{\text {uncorrected }}-\left(\frac{a}{N}+\frac{b}{N^{2}}\right)
$$

where a, b are free parameters that are estimated from fractions $\left(\frac{N}{2}\right.$ and $\left.\frac{N}{4}\right)$ of the data and $M I_{\text {corrected }}$ is the true calculated mutual information. Then, for each 'fly' we calculate the mutual information and average over them.

\subsection{Results}

\subsubsection{Information transmission under various system parameters without inhibition}

In the following, we will show the results without inhibition on Kenyon cells and mainly demonstrate that this leads to undesired and unrealistic characteristics. The value of the firing threshold of Kenyon cells in real cells is unknown. Therefore, all possible thresholds from 1 to 20 were studied. Similarly, little information about the exact connectivity between OPNs and Kenyon cells exists but studies have shown that this connectivity is small [Perez-Orive et al., 2002],[Honegger et al., 2011]. Thus, we also tested the system with different connectivity rates. Hence, mutual information between the environment and 10 or 20 Kenyon cells, respectively, was measured for all combinations of connectivity rate and firing threshold at low $\left(c_{\text {odor }}^{\text {low }}=0.15\right)$ as well as high $\left(c_{\text {odor }}^{\text {high }}=0.75\right)$ odor concentrations. First, for all results in this section it is assumed that there is no inhibition on the Kenyon cells. In the next section we investigate the inhibitory influences, too.

Fig. 3.3 (A,B) shows the dependency of mutual information for 10 as well as 20 Kenyon cells on the threshold and connectivity rate at low odor concentration

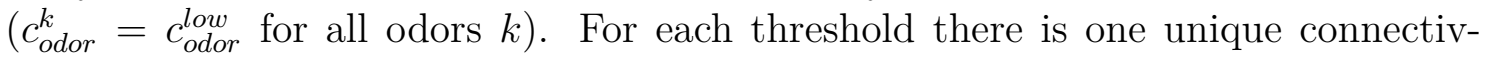
ity that corresponds to maximum mutual information. Another observation is the 
presence of an almost linear relation between connectivity rate and threshold for the maximal mutual information. Comparing the results for 10 and 20 Kenyon cells shows that the number of Kenyon cells does not qualitatively alter the results. A qualitatively similar behavior is observed for high odor concentration $\left(c_{\text {odor }}^{k}=c_{\text {odor }}^{\text {high }}\right)$. Hence, for each connectivity rate there is a shift to the right of the firing threshold that corresponds to maximum mutual information (Fig. 3.3 (C,D)).

As a low connectivity rate between antennal lobe and mushroom body $(r \approx 0.3$;

[Caron et al., 2013],[Butcher et al., 2012]) is assumed in flies, we show a cross-section of Fig. 3.3 (Fig. 3.4). The results clearly show a decrease in mutual information for high odor concentration and also a shift to higher threshold to obtain maximum mutual information as the firing rate becomes too high to enable a proper discrimination between odors. All these observations are in conflict with behavioral experiments where higher odor concentrations increase learning and memory efficiency, suggesting highly efficient information processing. Little is known with respect to plasticity of Kenyon cells and, thus, effective connectivity in this system might not change. The firing threshold, on the other hand, might well be a controllable parameter helping the insect when it encounters environments with different odor concentrations. However, such candidate mechanisms seems to be too slow (e.g., intrinsic plasticity; [Triesch, 2007], [Turrigiano, 2011] compared to behavioral time scales. Another option to control the efficiency of information transmission in a fluctuating odor concentration is the inhibition on Kenyon cells which will be investigated next.

\subsubsection{Information transmission under various system parameters with inhibition}

In the previous section we showed that the efficiency of information transmission depends critically on the odor concentration. However, the GABAergic inhibitory effect on Kenyon cells seems to have a critical role in sparse coding [Assisi et al., 2007]. As sparse coding is related to mutual information, we next analyze the role of inhibition on Kenyon cells in conjunction with the information transfer from the environment to the olfactory learning area in Drosophila.

Inhibition leads to an entirely different picture (Fig. 3.5): Fig. 3.5 illustrates the effect of inhibition on mutual information for different connectivity rates and thresholds when high odor concentration was presented to the system $\left(N_{K C}=10\right)$. Different strengths of inhibition (different $\alpha$ values) results in different levels of mutual information for different connectivity rates and thresholds. At connectivity rate equal to 0.3 (Fig. 3.6), most of the cases with inhibition are more effective in information transmission (higher mutual information) than without inhibition (dashed line). However, too strong inhibition leads to the effect that activities drop dramatically and, therefore, information transmission is decreased. Inhibition 
increases mutual information not only for one high odor concentration value: Fig. 3.7 shows the effect of inhibition on information transmission over different odor concentrations (averaged over all thresholds and $r=0.3$ ). For all concentration values the system with different inhibition strengths performs significantly better than without inhibition. Furthermore, the system does not show such a dramatic drop in information transmission increasing odor concentration. Thus, the olfactory system with feedback inhibition guarantees all the time an effective transmission of environmental information to the learning area when the animal moves towards the source of odor. 


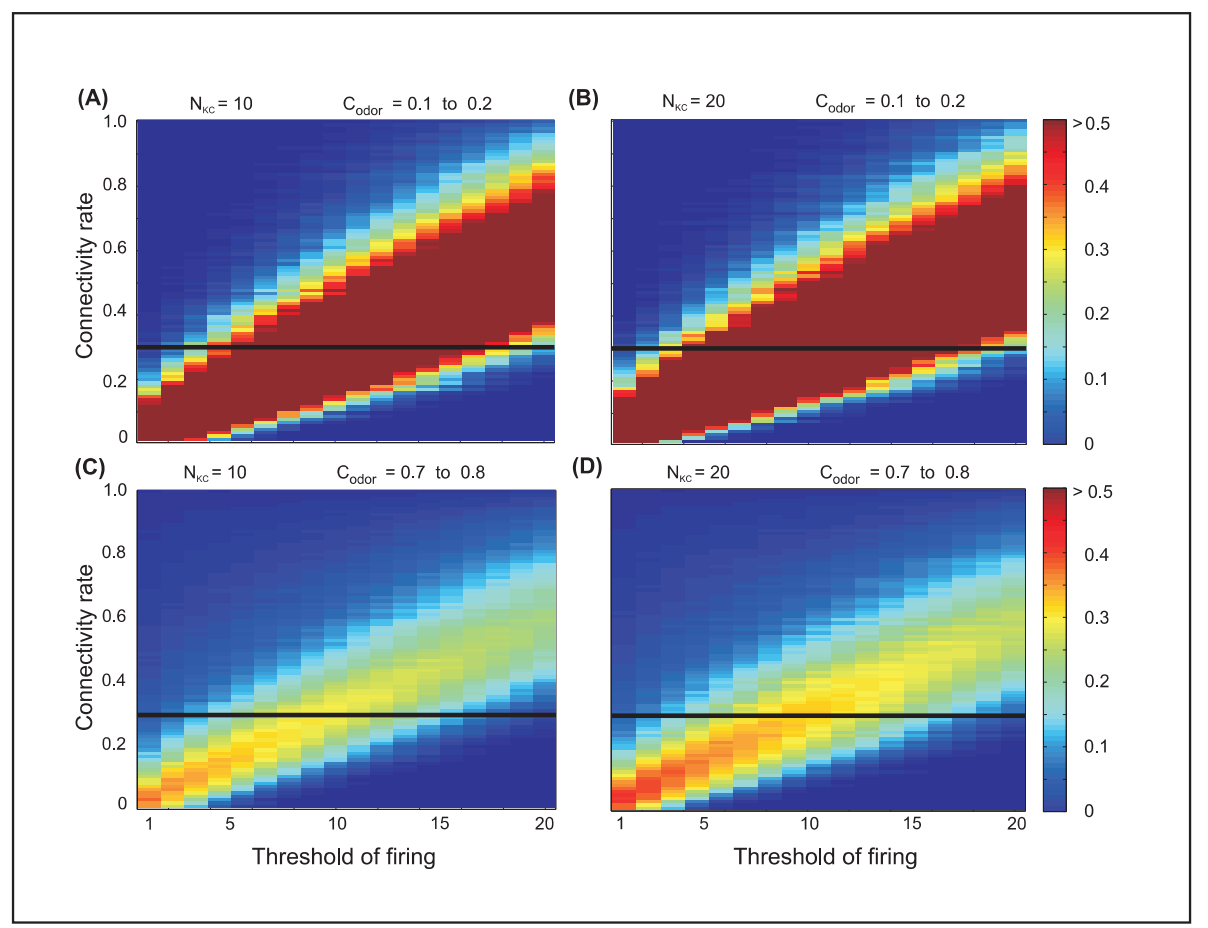

Figure 3.3: Mutual information between the environment (here 5 odors) and Kenyon cells for different firing thresholds of Kenyon cells and connectivity rate between the antennal lobe and the mushroom body in absence of inhibition on mushroom body. For each connectivity rate there is one unique threshold that corresponds to maximum mutual information. Moreover, increasing the threshold requires a higher connectivity rate to obtain maximum mutual information (thresholds between 1 to 20). The number of Kenyon cells does not alter the results qualitatively. (A-B) Low odor concentration $\left(c_{\text {odor }}^{\text {low }}=0.15\right)$. (C-D) High odor concentration $\left(c_{\text {odor }}^{\text {high }}=0.75\right)$ : for each connectivity rate and threshold of firing, mutual information is decreased compared to low odor concentration. Parameters: (A) $N_{K C}=10, c_{\text {odor }}^{\text {low }}=0.15$ (B) $N_{K C}=20, c_{\text {odor }}^{\text {low }}=0.15$ (C) $N_{K C}=10, c_{\text {odor }}^{\text {high }}=0.75$ (D) $N_{K C}=20, c_{\text {odor }}^{\text {high }}=0.75$. The horizontal lines indicate the connectivity rate equal to 0.3 (shown in Fig. 3.4) 


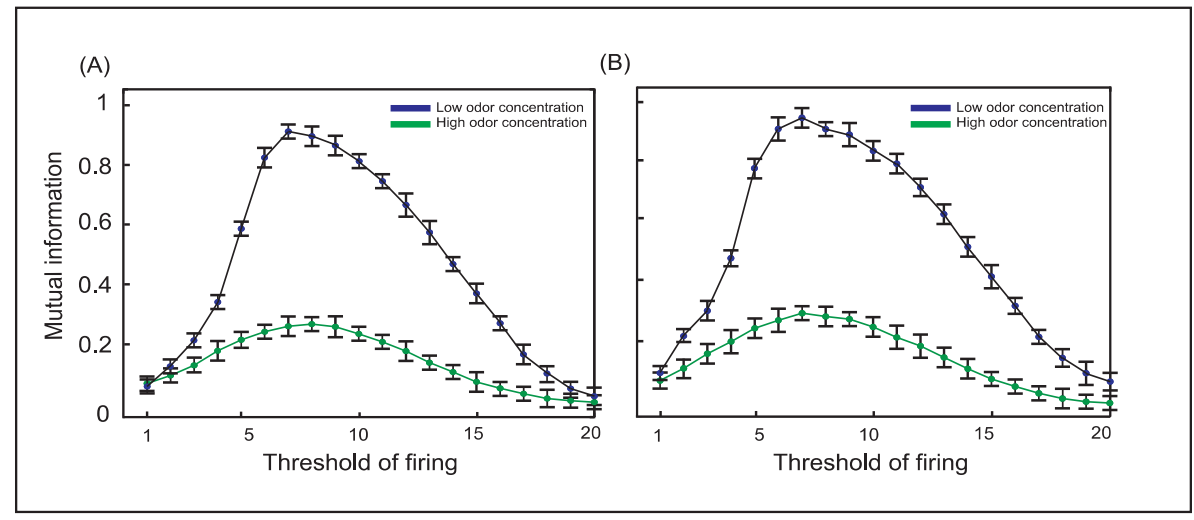

Figure 3.4: Mutual information between the environment and (A) 10 Kenyon cells or (B) 20 Kenyon cells for different thresholds. The connectivity rate set to 0.3 for both low and high odor concentrations. The maximum mutual information is obtained at threshold equal to 7 for low concentration and 8,7 when high odor concentration was presented to the neural system. For both levels of concentrations mutual information is decreased by increasing threshold. This clearly shows that an increase in odor concentration in absence of inhibition to Kenyon cells leads to a dramatic decrease in mutual information. 


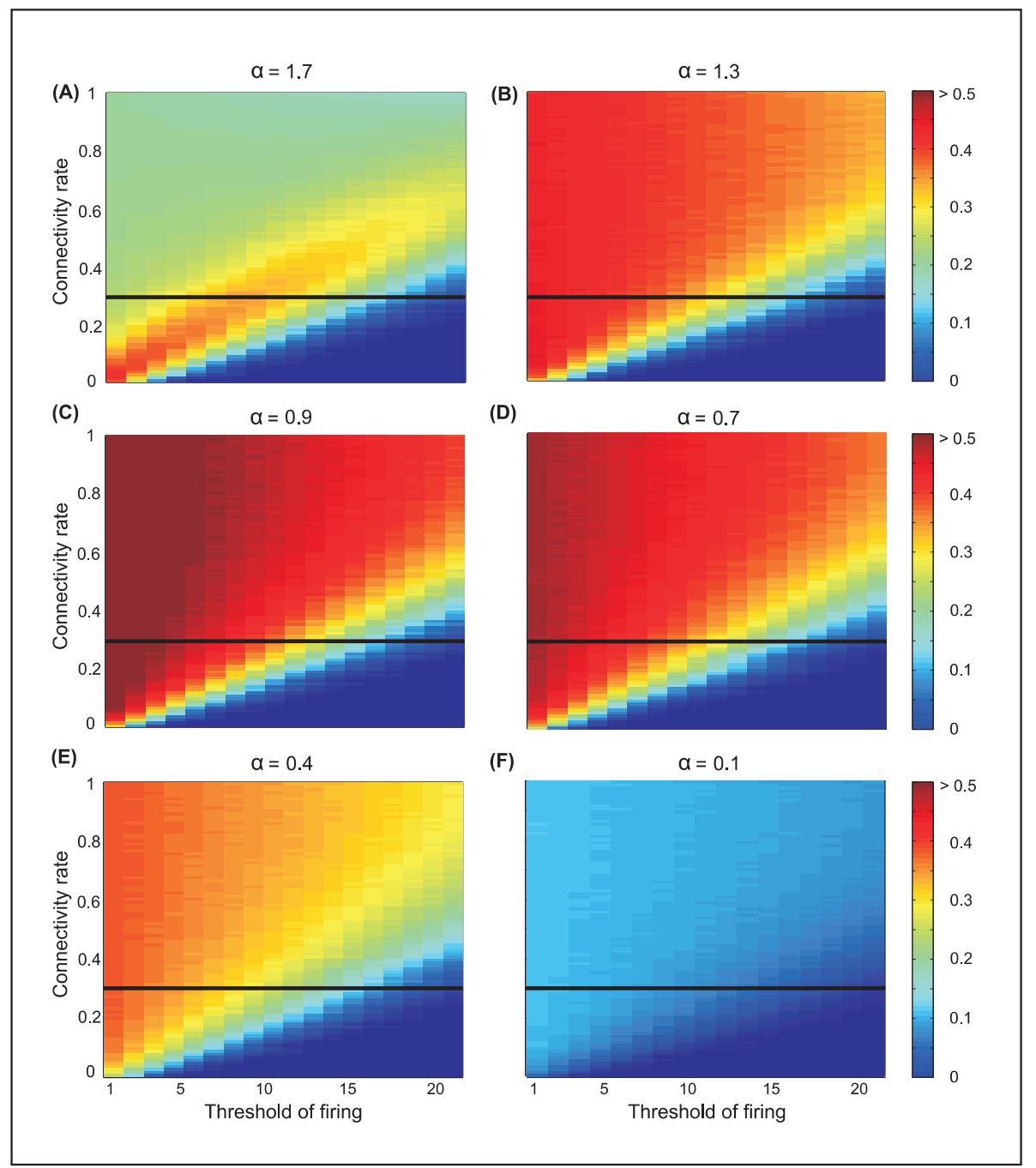

Figure 3.5: Mutual information between the environment (5 odors) and 10 Kenyon cells with the effect of inhibition on Kenyon cells and high odor concentration $c_{\text {odor }}^{\text {high }}=0.75$. The inhibition parameter $\alpha$ is equal to (A) $1.7,(\mathrm{~B}) 1.3,(\mathrm{C})$ 0.9, (D) 0.7, (E) 0.4, and (F) 0.1. Different parameter values yield different results on mutual information. The horizontal line shows the connectivity rate equal to 0.3 (shown in Fig. 3.6). 


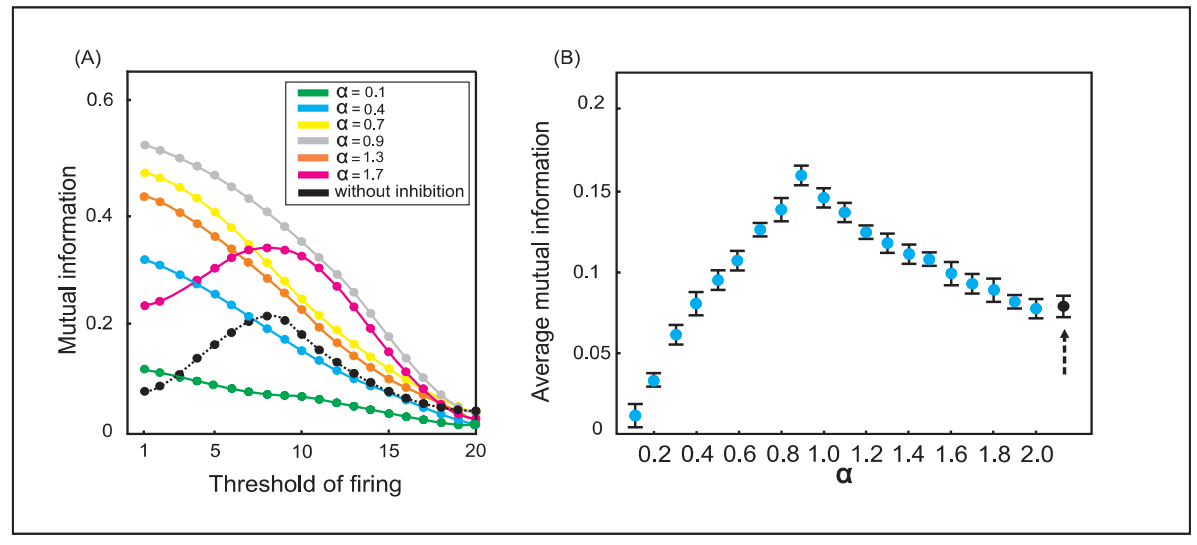

Figure 3.6: Mutual information between the mushroom body and the environment for connectivity rate equal to 0.3 for high odor concentration. (A) The mutual information is for most strengths of inhibition larger than without inhibition (dashed line). (B) Comparing the average mutual information with and without inhibition (black dot) shows that for most $\alpha$-values mutual information is higher than mutual information without inhibition.

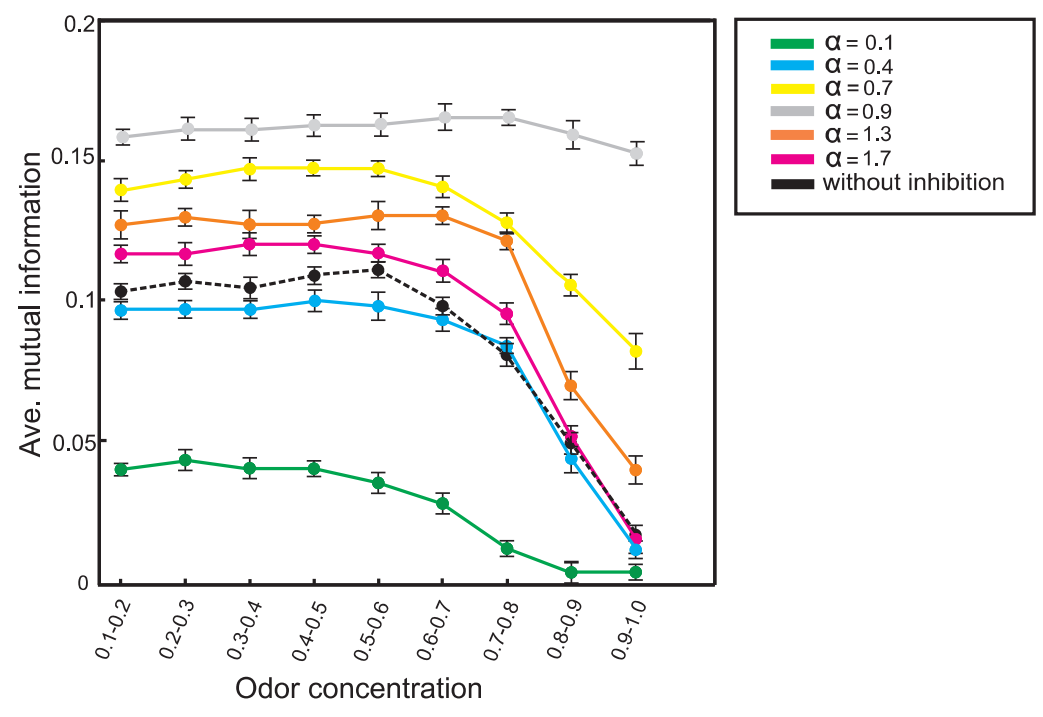

Figure 3.7: Mutual information between mushroom body $\left(N_{K C}=10\right)$ and the environment at connectivity rate equal to 0.3 and averaged over thresholds ( 1 to $20)$. Different odor concentrations were presented to the neural system with ( $\alpha=0.1$ to 1.7$)$ and without inhibition. Comparing mutual information for different $\alpha$ parameter values demonstrate that feedback inhibition increases mutual information for all concentrations. Furthermore, for several $\alpha$-values mutual information does not show such a dramatic drop in performance for increasing odor concentration. 


\subsection{Discussion}

Information theory has helped neuroscientists to study some structural and functional parameters that are difficult to assess by experiments and it offers some measures to evaluate information transfer by neural systems [Borst and Theunissen, 1999], [Dimitrov et al., 2011]. Furthermore, some tools from information theory are used to measure how much information a neural response contains about the stimulus in the environment and the statistical significance of variation in neural response for different stimulus intensities. The first attempts to apply information theory in neuroscience were to measure neural information flow in neural systems, as well as, the constraints that information theory imposes on the capability of neural system for communication. Thus, information theoretic measures have been used to infer the functional connectivity in neural systems [Singh and Lesica, 2010]. Another step was to discuss information as a constraint on neural system structure and function to optimize information transmission [Attneave, 1954]. This approach that the optimal information transfer guides the neuronal structure is still a very active field in neuroscience [Shlens et al., 2009] ,[Vanni and Rosenström, 2011].

In the current study, we have analyzed with tools from information theory the influence of olfactory system's parameter on information transmission between the environment and the mushroom body. The abstract model proposed in this study is capable of considering connectivity rate between antennal lobe and mushroom body, threshold of firing of neurons in the mushroom body, as well as the role of inhibition in different environmental situations that Drosophila melanogaster may encounter through its life time. For this purpose, we calculated mutual information between the environment composed of a set of odors with different levels of odor concentrations and subpopulation of neurons in the mushroom body. Comparing to other models of the (Drosophila) olfactory system [García-Sanchez and Huerta, 2003], [Huerta and Nowotny, 2009],[Huerta et al., 2004], our model does not assign an optimal value for threshold of firing in the Kenyon cells or an optimal connectivity rate between the antennal lobe and the mushroom body. It shows the presence of an almost linear relation between connectivity rate and threshold for the maximal mutual information.

We found that a plain feedforward system produces undesirable effects, like a drop in mutual information for increasing odor concentration. This is in contrast to behavioral studies [Masek and Heisenberg, 2008], [Yarali et al., 2009] which have demonstrated that higher odor concentration does not lead to a decrease in Drosophila efficiency for learning, memorizing, and discrimination of odors. Therefore, one would expect that the measure mutual information used in this study should be able to describe this phenomenon and, furthermore, predict the conditions necessary to obtain highest system efficiency. In absence of feedback inhibition, our simulations clearly assign a remarkable decrease in the system efficiency for odors with high concentrations. However, feedback inhibition of different strengths helps the system 
to obtain high system efficiency. Interestingly, a certain strength of inhibition (here equals to 0.9 ) results to the best performance. This inhibition strength guarantees a good system performance independent of the odor concentration. Hence, a pharmacological manipulation of this parameter in vivo should result to measurable changes in fly's behavior.

These findings support the idea of a key role of inhibition in keeping the system at or close to maximal mutual information or information transfer for all Kenyon cells (with varying connectivity rate) when the fly navigates in its natural environment, where odor concentration can very strongly vary. For instance, sensing very low odor concentration from a far distance navigating to it and eventually finding the source of odor (food or mate, for example) where the concentration has increased thousandfold. The importance of high and stable mutual information between a dynamic environment and the Kenyon cells becomes even clearer if we consider that the synapses between Kenyon cells and output neurons are very likely the place of association based learning [Séjourné et al., 2011]. 



\section{A retrograde signaling model of information processing in the Drosophila olfactory learning}

\subsection{Introduction}

Behavioral processes including associative learning allow animals to cope with a changing environment where they search for their needs while avoiding different kind of dangers. Insects are evolutionary successful animals that their small brains have provided intelligent solutions to wide range of problems in order to live in changing environments. Studies on insects' brain assume that simple neural circuits are responsible for different behaviors. Flies have small brains, but exhibit complex learning behaviors. The limited number of neurons in their brains allows the analysis of neural functions. Hence, the Drosophila olfactory system can serve as a useful model to study cognitive processes to search for their neural circuits as well as the cell and molecular mechanisms underlying different behaviors [Kay and Stopfer, 2006]. Moreover, the availability of manipulation by transgenic technology has made the Drosophila olfactory system a powerful tool for examining hypotheses of neuronal mechanisms of learning [Keene and Waddell, 2007],[Mcguire et al., 2005]. In addition, Drosophila's brain has been used as a source of inspiration for robotic architectures [Arena et al., 2010].

Second order conditioning originally described by Pavlov have been studied by psychologists for decades [Miller et al., 1995] and have been conducted in many insects species like honeybees [Hussaini et al., 2007b],[Laloi et al., 1999] and crickets [Mizunami et al., 2009]. Although the Drosophila olfactory system has provided basic understanding of the cellular and molecular mechanism underlying first order 
conditioning as simple associations of a given odor with electric shock as punishment, however, much less emphasis has been placed on second order conditioning. Recently, second order conditioning in the Drosophila olfactory system has been demonstrated [Tabone and Belle, 2011] which presents an opportunity for assessing the role of genes and neural networks in learning and memory, including second order conditioning. In addition, modern neuroscience has recently focused on second order conditioning as a means for exploring the mechanism of information processing in health and disease [Gewirtz, 2000].

Second order conditioning is a form of higher cognition wherein a previously Conditioned Stimulus can be associated with a second different stimulus to elicit a conditioned response. Initial training involves the pairing of the first odor with electric shock (first order conditioning session) followed by second order conditioning session in which the first odor is paired subsequently with the second odor. If second order conditioning is performed successfully, flies will demonstrate a conditioned response with the second odor. It has been demonstrated that in crickets, second order conditioning requires neurotransmitter release from dopaminergic neurons [Mizunami et al., 2009]. This observation has not been reported in the Drosophila olfactory system yet. Hence, we developed a model with different assumptions which is a retrograde signaling based model for second order conditioning in the Drosophila olfactory system.

Over several decades, it has become clear that information exchange in neural systems is bidirectional: the postsynaptic cell provides a variety of retrograde signals to the presynaptic neuron in response to the presynaptic activity (spiking). This interaction is crucial for the formation and plasticity of neural connections [Regehr et al., 2009],[Tao and Poo, 2001]. One of the well known retrograde signals which has been studied in the neurons of higher animals is nitric oxide [Yan et al., 2012], [Steinert et al., 2010], [Luo and Zhu, 2011](Fig. 4.1).

Our model integrates is based on hypothetical mechanisms of retrograde signaling in Drosophila olfactory system and Hebbian learning rule for synaptic plasticity at the neural network level to describe olfactory conditioning behavior in flies (Fig. 4.2). Another feature of this model is that both candidate places for associative learning are involved in the modeling of learning processes. 


\section{Cellular mechanism of Nitric Oxide}

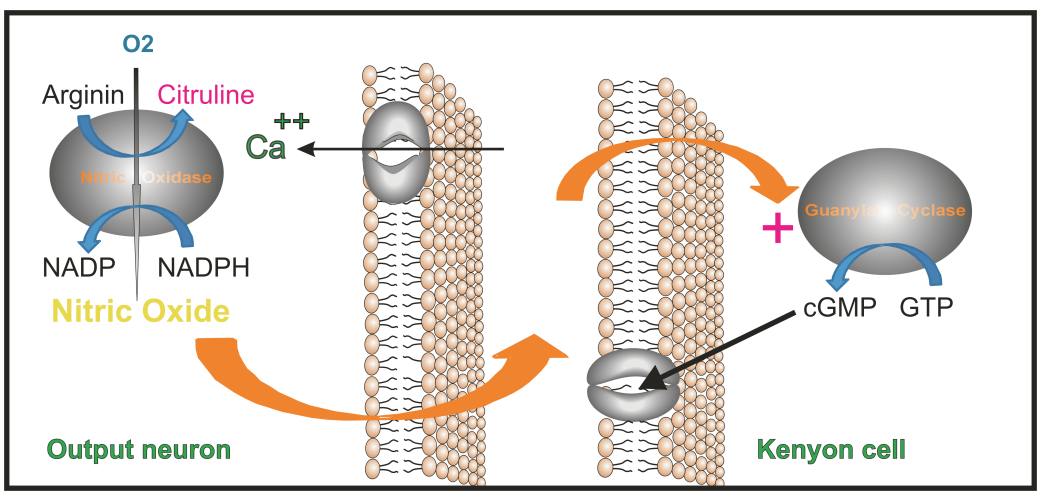

Figure 4.1: The cellular mechanism of affecting the synaptic efficacy by nitric oxide. Nitric oxide is produced as a consequence of spiking of the Kenyon cells. Nitric oxide diffuses into all Kenyon cells synapses and may increase the cGMP level of Kenyon cells which consequently increases intercellular calcium level by different mechanisms. This may eventually affect the synapses.

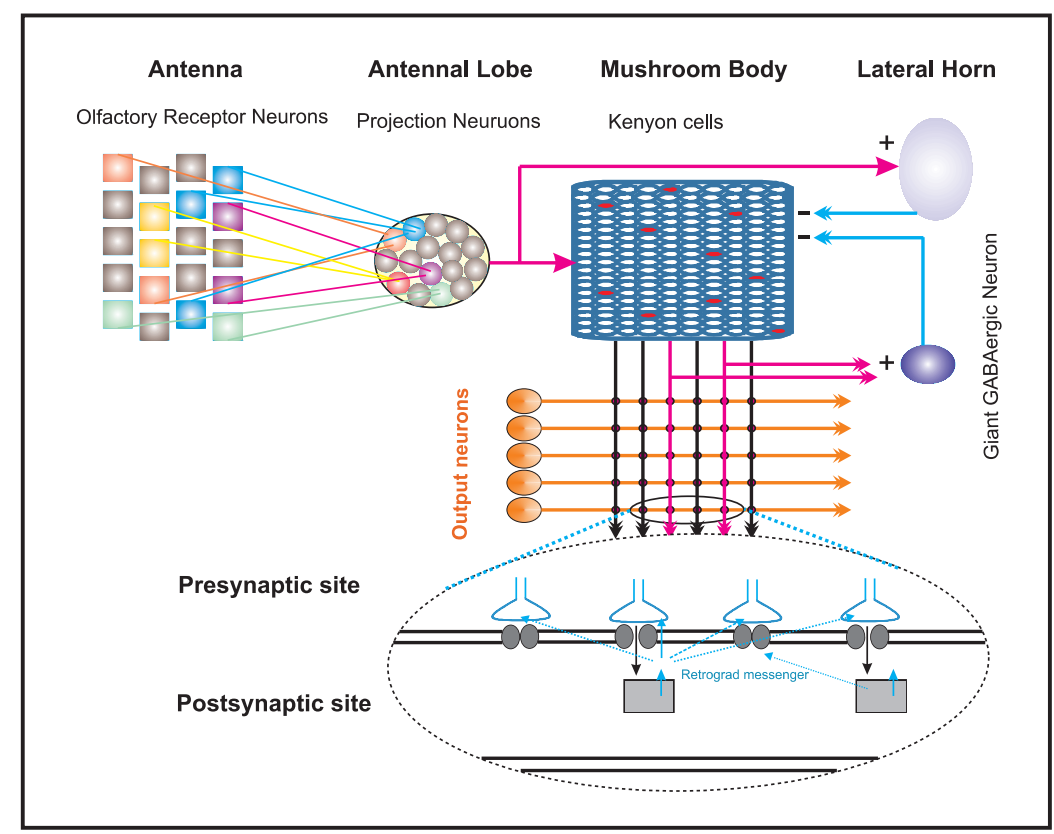

Figure 4.2: The retrograde signaling hypothesis. Odor activates a subset of the Kenyon cells. Their sparse spiking activates production of the retrograde signal. The produced retrograde signal in the output neuron diffuses into the presynaptic neurons as a function of distance between the synapse and the production site. 


\subsection{Methods}

In this section, the structure of the neural model as a simplified olfactory system of Drosophila, the neural spiking equations used to simulate neural activity, hypothetical molecular mechanisms underlying the change of synaptic efficacy and the protocol of simulations of olfactory conditioning in a two dimensional space are described.

\subsubsection{Insilico-fly}

To develop a model of olfactory conditioning, a two dimensional space was defined in which the fly navigates to search for food. The motion of the fly was modeled as a random walk in this space where each step of the fly's motion takes one second. In each step, the distance of the insilico-fly and the food source is calculated, if the distance of the fly and the food source is less than a given threshold then we assume that the concentration of odor is high enough to stimulate the Drosophila olfactory system. In this situation, electric shock may also be presented to the fly 0.05 second after odor presentation. This paradigm is used in all simulations presented here. The fly that navigates the two-dimensional space is called 'insilico-fly' because its general structure is based on the experimental data of the Drosophila olfactory system.

\subsubsection{Architecture of the olfactory system}

The olfactory system of the insilico-fly is composed of the antennal lobe (50 projection neurons), the mushroom body (2000 Kenyon cells), one output neuron, one inhibitory neuron and one dopaminergic neuron. To model sparse coding in the mushroom body, very low connectivity rate between the mushroom body and the antennal lobe was used as random values between 0.05 and 0.3 . The connectivity rate is defined as the average percentage of random connection of each the projection neuron in the antennal lobe to the Kenyon cells in the mushroom body. The matrix of connectivity between the projection neurons and the Kenyon cells is constructed and fixed to use in all odor presentations in the simulations. This is crucial to have insilico-fly with determined neural structure to obtain reliable results in all simulations when different odors are presented to the fly.

The dopaminergic neuron generates spikes with firing rate equal to 0.5 as a consequence of a second shock presentation. An inhibitory neuron is fully connected to the Kenyon cells which may inhibit the spiking of Kenyon cells in each time bin (equal to 10 millisecond). The inhibitory neuron control the firing rate of the Kenyon cells such that it plays critical role through learning process to control synaptic efficacy and the retrograde signal production. The output neuron serves as motor neuron 
such that its firing rate determines the avoidance behavior of the insilico-fly. If the firing rate exceeds a threshold equal to 0.8, then the insilico-fly will avoid the given odor. Each Kenyon cell has one synapse with the output neuron such that each pair of adjacent synapse has equal distances. Therefore, the connection of the mushroom body and the output neuron is represented as a linear 2000 synapses with equal distances.

\subsubsection{Neuronal activity models}

An odor as a conditioned stimulus is presented to the insilico-fly for a second when the fly is close enough to food source. This may activate a set of the projection neurons (here 20 projection neurons of total 50 projection neurons) in the antennal lobe to generate spikes in each time bin which are equal to 10 milliseconds. The probability of generating a spike in each time bin is determined by firing rate of the projection neurons which was randomly selected from values between 0.1 and 0.2.

Different odors activate different sets of the projection neurons in the antennal lobe. All these strategies are used in order to obtain minimum overlap in the number of activated Kenyon cells by the first and the second odor presentations during conditioning processes. The neuron model used for the Kenyon cells and the output neuron in all simulation are an 'Integrate and Fire' neuron model (equation 4.1). The input to each Kenyon cell from the antennal lobe may have different number and intensity. Therefore, different activated Kenyon cells may generate spikes with different frequency.

$$
C \frac{d V}{d t}=-g_{\text {leak }}\left(V-V_{\text {rest }}\right)+I(t)
$$

The parameter values that were used in the 'Integrate and Fire' neuron model are the electrophysiology data on honeybees [Wüstenberg et al., 2004] which is listed in Table 1 .

Table 4.1: Parameters of the Integrate and Fire neuron model

\begin{tabular}{crr}
\hline \hline$V_{\text {rest }}$ & Resting potential & $-84 \mathrm{mV}$ \\
$V_{\text {thresh }}$ & Threshold of spiking & $-25.8 \mathrm{mV}$ \\
$V_{\text {recov }}$ & Recovery threshold & $-40.2 \mathrm{mV}$ \\
$V_{\text {spike }}$ & Spike potential & $9.5 \mathrm{mV}$ \\
$g_{\text {leak }}$ & Membrane conductance & $0.26 \mathrm{nS}$ \\
$C$ & Membrane capcitance & $0.26 \mathrm{nS}$ \\
\hline
\end{tabular}


The spiking of each neuron results in a current into the post-synaptic neuron that is expressed as equation 4.2 :

$$
I(t)=\omega \frac{t}{\tau} e^{\frac{-t}{\tau}} \sum_{t p} \delta(t-t p)
$$

where $\tau=0.3$ second and $\omega$ is synaptic weight.

Dopamine has been suggested to mediate reinforcing properties of aversive unconditioned stimulus [Schwaerzel et al., 2003], [Riemensperger et al., 2005]. Electric shock is presented as an unconditioned stimulus which elicits dopaminergic neuron to release dopamine. Dopamine is released non-specifically to all Kenyon cells. The dynamics of dopamine available in extra-cellular space of the Kenyon cells are modeled as expressed by equation 4.3 [Izhikevich, 2007].

$$
\dot{d}=\frac{-1}{\tau_{d}}\left(d+\sum_{t d} \delta(t-t d)\right)
$$

where $\tau_{d}=0.1$ second

The firing rate of one dopamienrgic neuron and released dopamine in extra-cellular gap between the dopaminergic neurons and the Kenyon cells is shown in (Fig. 4.3). The released dopamine is removed from the gap by some mechanisms. In absence of the electric shock presentation, a basal level of dopamine released by dopaminergic neuron is assumed to be equal to 0.05 . 
4.2 Methods

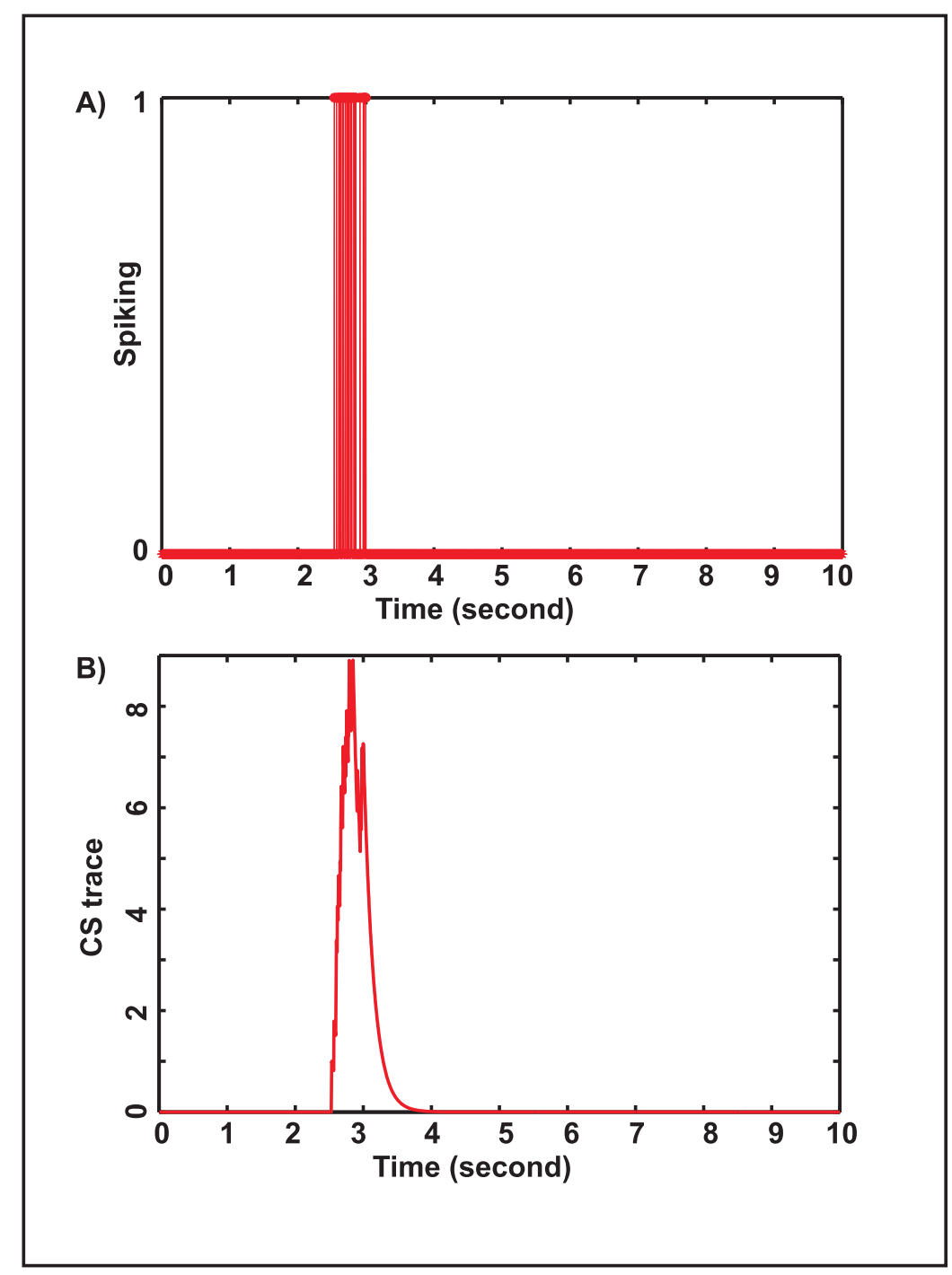

Figure 4.3: Unconditioned Stimulus presentation. A) Simulation of the dopaminergic neurons' activity in response to a second electric shock. B) Unconditioned Stimulus trace as the extracellular released dopamine by dopaminergic neuron. Released dopamine is degraded by some mechanism so its amount decays rapidly to zero. 
To assess the influence of different inhibitory strengths on the mushroom body, we introduce a probability $p_{I}$ that Kenyon cells spiking at each time bin can be inhibited. This probability depends on the average activity in the mushroom body $\tilde{\chi}$ without inhibition (equation 4.4).

$$
p_{I}=e^{-\frac{\alpha}{\tilde{\chi}}}
$$

The parameter $\alpha$ (between 0.05 and 1.75) determines the different strengths of inhibition. In this model, we used optimal inhibition parameter (equal to 0.9) determined by information theoretic model of the (Drosophila) olfactory system presented in chapter 3.

\subsubsection{Retrograde signaling production and diffusion}

In this model, it is assumed that some kind of chemicals as the retrograde signals is produced in the output neuron which is triggered by the spiking of Kenyon cells. The amount of retrograde signal production is assumed to be the function of input current to the output neuron (equation 4.5).

$$
R_{i}(t)=\frac{1}{\sigma}\left(e^{-\alpha \cdot t}-e^{-\beta \cdot t}\right) \sum I_{i}(t)
$$

where $R_{i}$ is produced retrograde signal by the output neuron induced by $K C_{i}$. $\sigma=100, \alpha=0.08, \beta=100$.

The produced retrograde signal diffuses into all synapses. The amount of the received retrograde signal is assumed to be the function of half-time of retrograde signal and the distance between each synapse and the retrograde signal production site (equation 4.6).

$$
\left[R_{i}\right]_{x}=\left[R_{i}\right]_{0} e^{-\ln x}
$$

where $\left[R_{i}\right]_{0}$ is the total amount of the produced retrograde signal in an odor presentation in each second, $x$ is the distance of synapses and the retrograde signal production site, $D=0.001$ is a constant of retrograde signal.

The production and decay of the retrograde signal at the production site has been shown in (Fig. 4.4). 


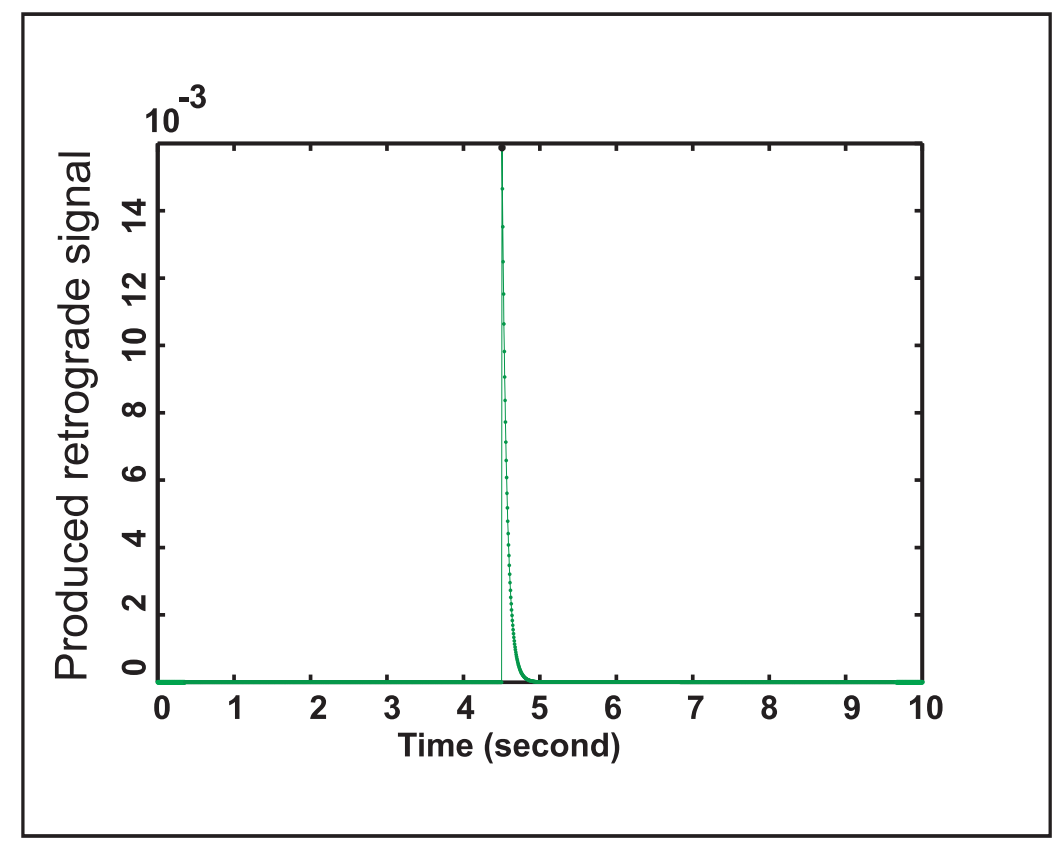

Figure 4.4: Production of the retrograde signal. The retrograde signal is produced as a consequence of a second spiking of the activated Kenyon cells. The retrograde signal diffuses rapidly from the production site to the Kenyon cells' synapses.

\subsubsection{Synaptic efficacy between the antennal lobe and the mushroom body}

The diffused retrograde signal is received by the Kenyon cells according to the distance of synapses and production sites. The total amount of received retrograde signal by each Kenyon cell determines the level of changes in the synaptic weight between the activated Kenyon cell and the activated projection neurons. To model this effect, we used a sigmodial function to obtain high levels of change in the synaptic weight for very high levels of received retrograde signal (equation 4.7).

$$
\omega_{1}=\frac{\left(\sum_{j=1}^{N_{K C}} R_{1, j}\right)^{2}}{1+\left(\sum_{j=1}^{N_{K C}} R_{1, j}\right)^{2}}
$$

Where $R_{1, j}$ is the total amount of the received retrograde signal by a Kenyon cell synapse.

$N_{K C}$ is the number of total Kenyon cells (here 2000) and $\omega_{1}$ is the synaptic efficacy between a Kenyon cell and the activated projection neurons. It is important to notice that the amount of the produced retrograde signal depends on the synaptic 
weight between the mushroom body and the output neuron as well as the firing rate of the Kenyon cell. So it can be indirectly affected by odor-shock pairing.

At the end of each trial of odor-shock pairing, the synaptic efficacy between the Kenyon cells and the output neuron are updated using equation 4.7 and also the received retrograde signal in all Kenyon cells are fully removed. This prevents the accumulation of retrograde signal in the Kenyon cells after a few trials. In the beginning of any trial of pairing, the updated synaptic efficacy is used and at the end of the trial the synaptic efficacy between the Kenyon cells and the output neuron are updated again.

\subsubsection{Dynamics of the synaptic efficacy between the mushroom body and the output neuron.}

The dynamics of the synaptic weight between the mushroom body and the output neuron was modeled based on [Izhikevich, 2007]. We used a simple phenomenological model for changing the synaptic weights that captures odor and electric shock association.

$$
\dot{C}=\frac{-1}{\tau_{c}}\left(C+\Delta \sum_{t c} \delta(t-t c)\right) ; C \geq 0
$$

where $\tau_{c}=0.2$ seconds

$$
\dot{\omega}_{2}=C d
$$

Where $\omega_{2}$ denotes for the synaptic weight between a Kenyon cell and the output neuron, $C$ for odor trace which is assumed to be the activation of enzyme important for plastcity.

$D$ describes the extra-cellular concentration of dopamine and $\delta(t)$ is the Dirac function that step-increases the variable $C$.

We have modified the model of [Izhikevich, 2007] by replacing Spike Time Dependent Plasticity rule with a simple Hebbian rule defined in Table 4.2.

Table 4.2: Hebbian learning rule used in the model

\begin{tabular}{crrrr}
\hline \hline Kenyon cell & 1 & 1 & 0 & 0 \\
output neuron & 1 & 0 & 1 & 0 \\
\hline$\Delta$ & 1 & -1 & -1 & 0
\end{tabular}

The Hebbian rule in Table 4.2 suggests that high firing rate of the Kenyon cells in pair with high firing rate of the output neuron leads to the strengthening of the synaptic weight between the activated Kenyon cells and the output neuron. 


\subsubsection{Simulation studies}

To simulate first and second-order conditioning in the insilico-fly with the developed model of the Drosophila olfactory system, the protocol of training the fly was used [Tabone and Belle, 2011]. The hypothetical mechanism underlying the cellular and network mechanisms of olfactory learning in the in-silico fly are illustrated in Fig.4.5 to Fig. 4.7. This is categorized as three phases, in each phase, the cellular and network mechanisms are discussed.

\section{First phase: CS1 and electric shock pairing}

In the first phase of the simulations, the first odor as conditioned stimulus and electric shock as unconditioned stimulus are presented to the insilico-fly simultaneously for 10 trials. Through learning, the stimulated projection neurons by the first odor activate small populations of the Kenyon cells in the mushroom body which consequently results in an increase in the synaptic weight between the mushroom body and the output neuron as well as the production and diffusion of retrograde signal into all Kenyon cells. The diffused retrograde signal increases the weight between the synapses of activated Kenyon cells and the activated projection neurons in the antennal lobe. This lead to more strengthening of synapse between the Kenyon cells and the output neuron according to equations 4.8 and 4.9 (Fig. 4.5). 


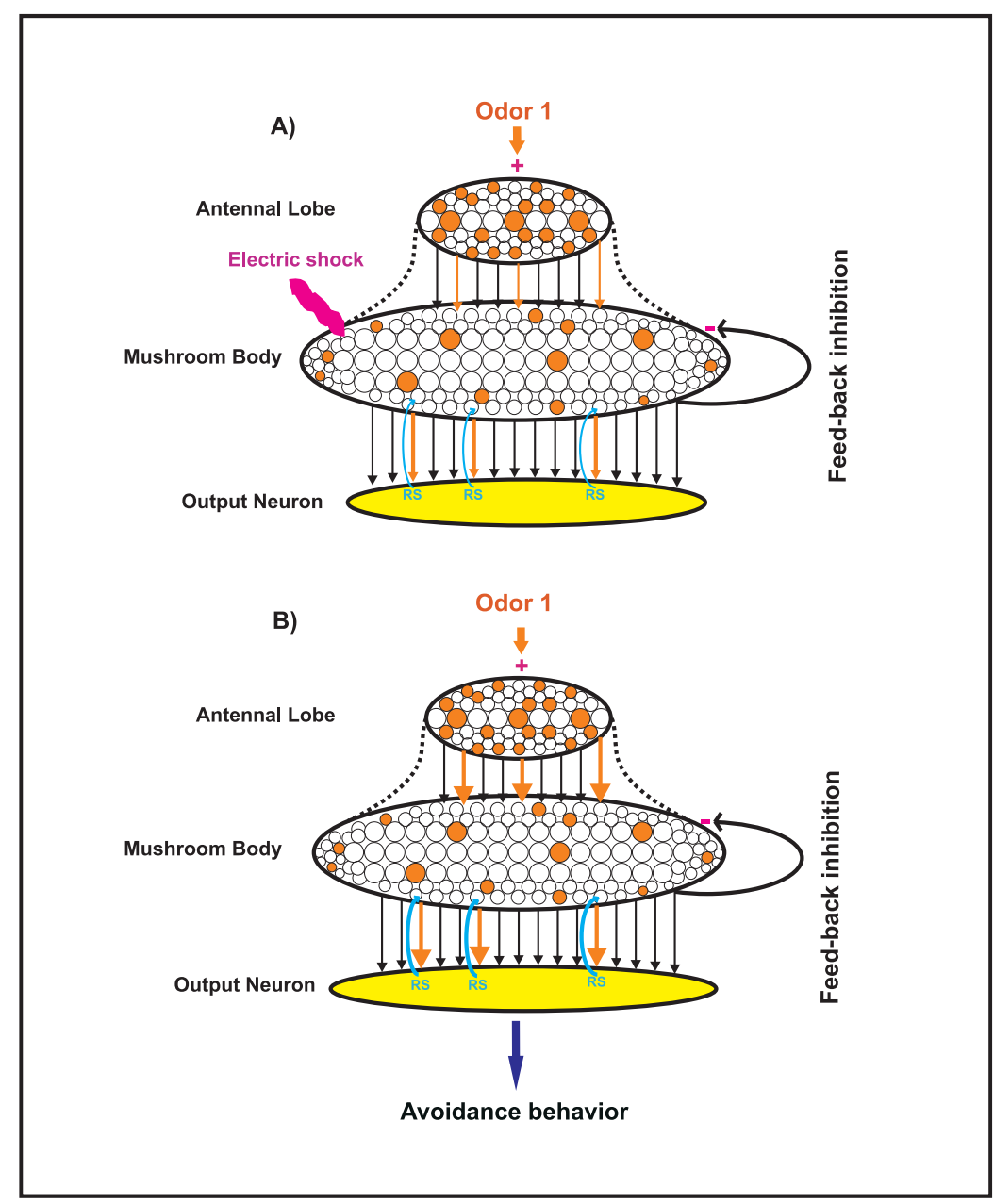

Figure 4.5: The neural events underlying the first phase of simulations. A) The first odor as conditioned stimulus is paired with the electric shock as unconditioned stimulus. This leads to strengthening of the synaptic weight between activated the Kenyon cells in the mushroom body and the output neuron. B) High amounts of the retrograde signal diffuses into the mushroom body and affect the synaptic weight between the activated Kenyon cells and the activated projection neurons in the antennal lobe. This leads to an increase in firing rate of the output neuron which triggers avoidance behavior if exceeds a threshold. 


\section{Second phase: CS1 and CS2 pairing}

After performing the first phase; the trained odor is paired with the second odor which activates a different sets of the projection neurons in the antennal lobe. In the beginning of this phase, the synapses between the activated Kenyon cells by the first odor and the output neuron is strong enough to produce and release of the retrograde signal, while the synapses between the activated Kenyon cells by the second odor and the output neuron is weak. According to the distance of synapses and the retrograde signal production sites, each activated Kenyon cell by the second odor may receive enough retrograde signal to change the synaptic weight between activated projection neurons and the activated Kenyon cells by the second odor. This leads to an increase in firing rate of the activated Kenyon cells by the second odor which consequently increases the synaptic weight between the activated Kenyon cells and the output neuron (equations 4.8 and 4.9). Briefly, at the end of the pairing of the first odor and the second odor the synaptic weight between responding projection neurons and the Kenyon cells as well as synapses between the responding Kenyon cells and the output neuron have raised enough to trigger the output neuron to fire at high rate (Fig. 4.6). 


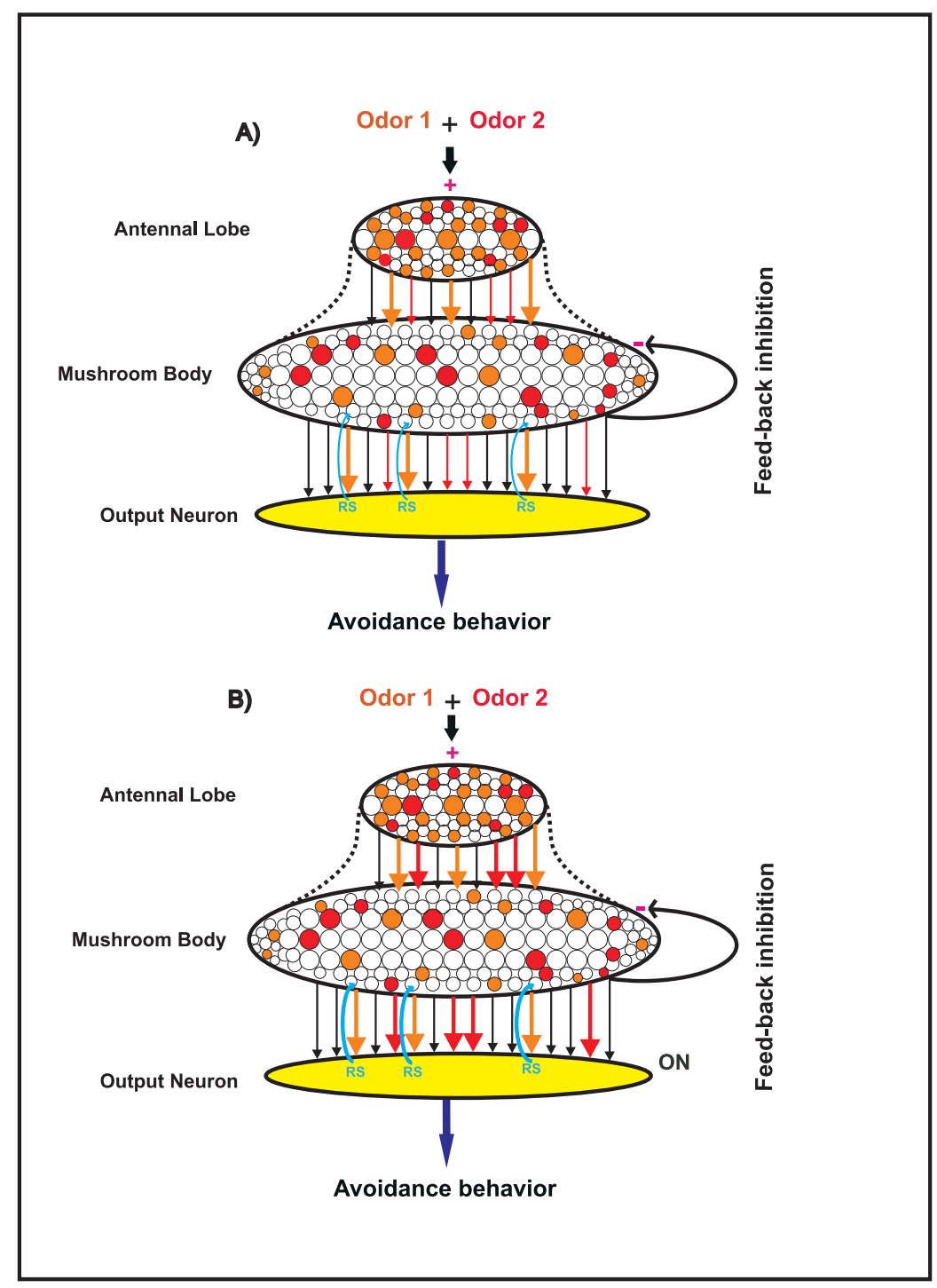

Figure 4.6: The neural events underlying the second phase of simulations. A) The second odor is paired with the first odor after (trained odor). At the beggining of the pairing, high amounts of the retrograde signal diffuses from the output neuron to the mushroom body. B) After a few trials the synaptic weight between the activated projection neurons by the second odor in the antennal lobe and the Kenyon cells in the mushroom body is increased. This results in an increase in the firing rate of the activated Kenyon cells by the second odor. This eventually results in an increase in the synaptic weight between the mushroom body and the output neuron for the activated Kenyon cells by the second odor. 


\section{Third phase: CS2 presentation}

To test whether the second odor has produced the capability to stimulate the output neuron to fire at high rate, the responding projection neurons to the second odor are being activated just for a few time to observe its impact on the output neuron firing rate (Fig. 4.7). In addition, the updated weight of synapses between the projection neurons and the Kenyon cells are set to their basal level $(=0.5)$ see its impact on the output neuron's firing rate. For this purpose, two cases are considered; one with the presence of the retrograde signaling, another, without the diffusion of the retrograde signaling in response to the odor presentation.

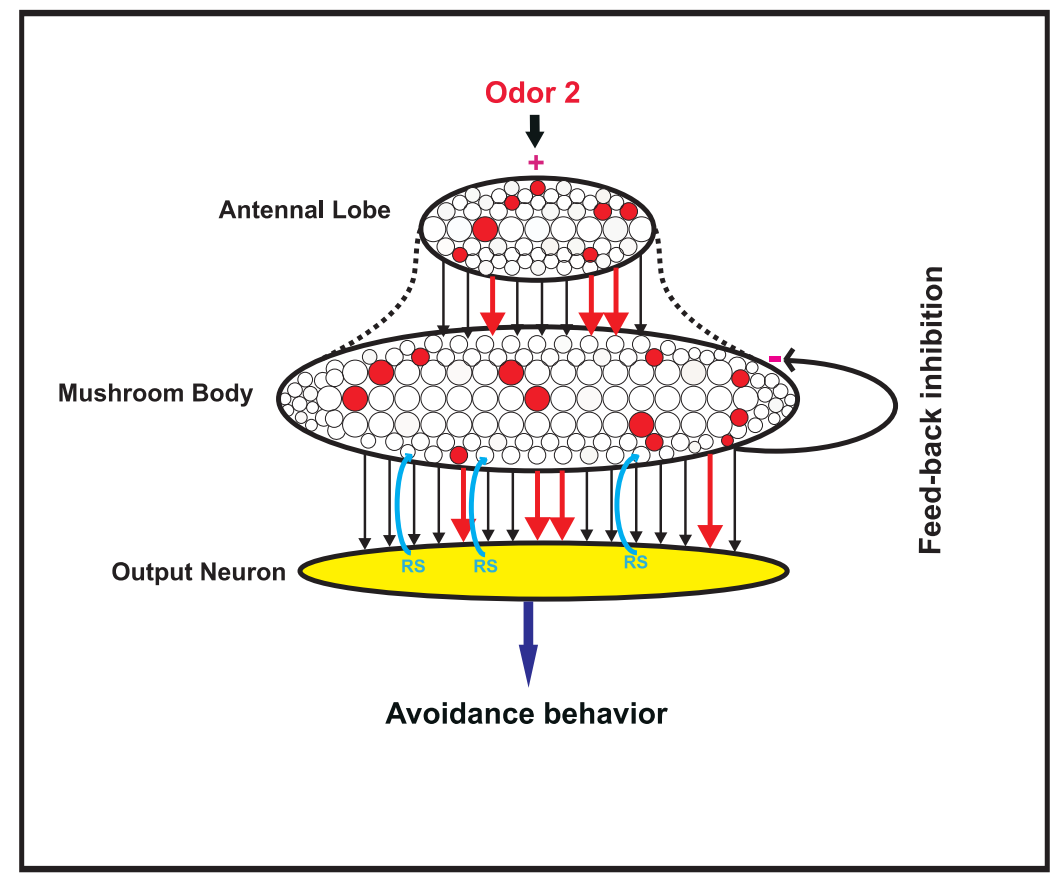

Figure 4.7: The neural events underlying the third phase of simulations. After pairing of the first and the second odor, the second odor is presented to the neural system to test its ability to trigger the output neuron to fire at high rate. The synaptic weight between the activated Kenyon cells and the projection neurons has been strengthened due to the diffused retrograde signal. This has caused indirectly the increase in the synaptic weight between the activated Kenyon cells and the output neuron. Feedback inhibition controls the firing rate of the Kenyon cells which may be at high rate at the end of the pairings. 


\section{Olfactory conditioning in two-dimensional navigation of fly}

To show the process of olfactory conditioning, we considered a two dimensional space in which the food resource and the insilico-fly are located at the separated places. The fly starts a random walk to find the food resource. If the fly is close enough to the food, then odor and the electric shock or both, are presented to the fly. In the second simulation, both the first odor (from previous simulation) and the second odor are located close to each other and are presented to the fly through its navigation. In the third simulation, the second odor (trained odor) in second phase is presented individually to the insilico-fly to study the behavior of the fly.

\subsection{Results}

In this section, the results for each phase of association learning in the insilico-fly is presented separately. The simulations of two-dimensional navigation of fly for each phase are presented as well.

\subsubsection{First phase}

The first odor was presented to the insilico-fly which stimulates a determined set of the projection neuron in the antennal lobe. The activated projection neurons trigger some Kenyon cells in the mushroom body to spike with different rates. The variation in firing rate of the Kenyon cells is due to the different number of the inputs to the different Kenyon cells. The activated Kenyon cells by the first odor as well as their firing rate for the first 200 Kenyon cells in the mushroom body is shown in (Fig. 4.8). The activated Kenyon cells trigger the output neuron to produce different amounts of the retrograde signal that are received by all Kenyon cells according to the distance of synapses and the production sites. The amounts of the received retrograde signal by each Kenyon cell is shown in Fig. 4.8 B. It also illustrates that at the end of the trials non-spiking Kenyon cells may receive different levels of the retrograde signal, diffused by the activated Kenyon cells by the first odor. 
A)

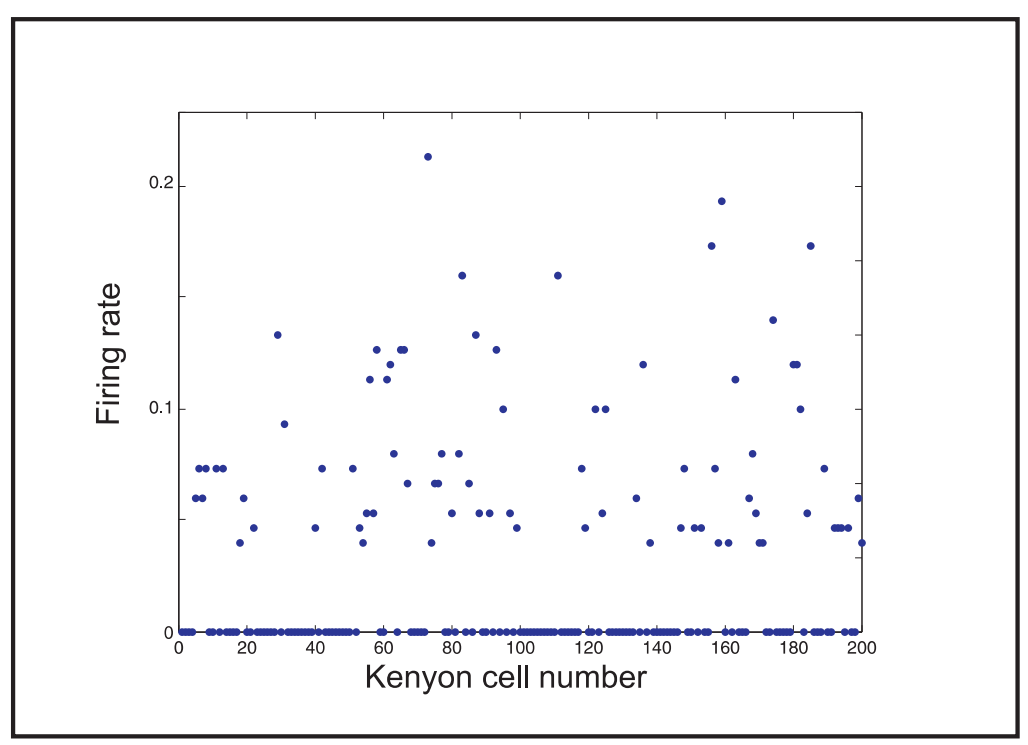

B)

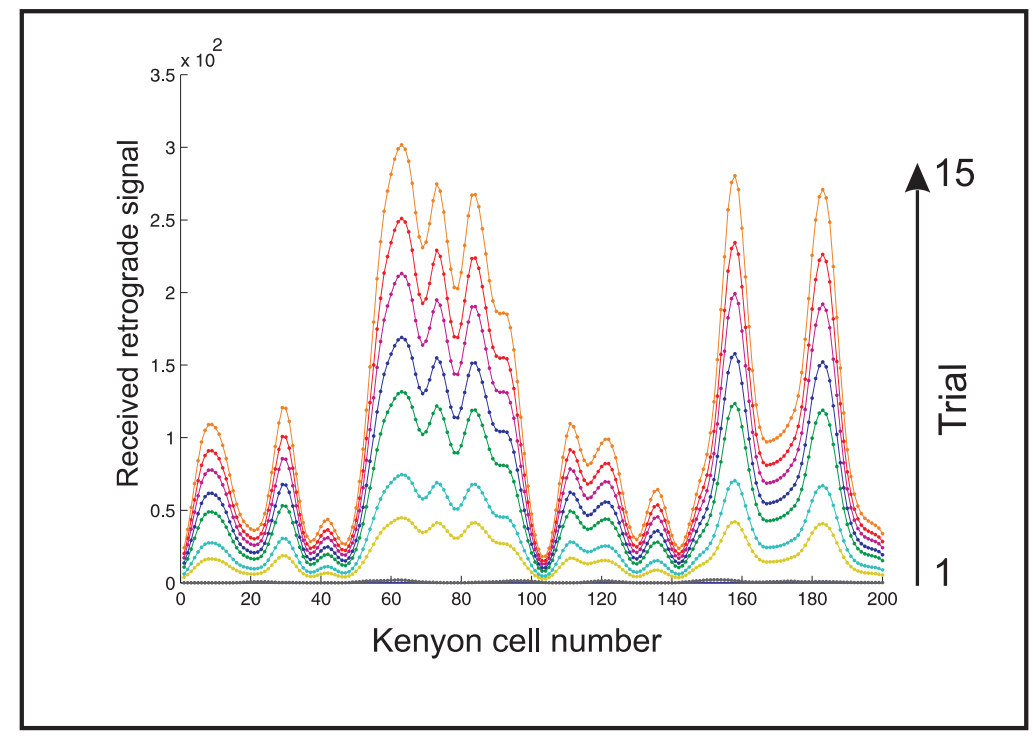

Figure 4.8: A) The firing rate of the activated Kenyon cells by the first odor. The activated Kenyon cells by the first odor may spike with different rates. B) The activated Kenyon cells trigger the output neuron to produce the retrograde signal that diffuses into all Kenyon cells. The amount of the received retrograde signal by each synapse depends on their distance from the production sites of the retrograde signal. The non-spiking Kenyon cells may receive different levels of the retrograde signal. 
In each trial of the first odor and shock pairing, the synaptic weight between the activated Kenyon cells by the first odor and the output neuron is strengthened according to the equation 4.8 and 4.9. The change of the synaptic weight between the activated Kenyon cells by the first odor and the output neuron for different trials is shown in Fig. 4.9. The change of synaptic weight is due to both the first odor and shock pairing as well as Hebbian rule used in the model. Non-spiking Kenyon cells show no change in their weight.

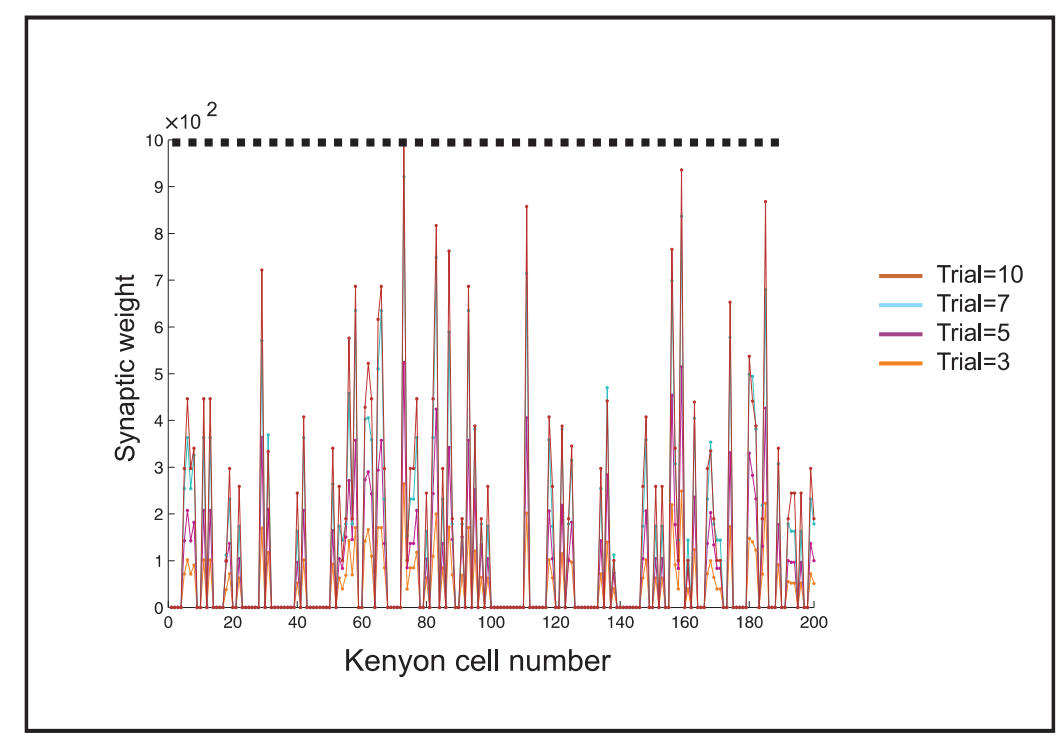

Figure 4.9: The change of synaptic weight between the Kenyon cells and the output neuron for different trials of the first odor and shock pairing. The change of synaptic weight depends on both the first odor and shock pairing and Hebbian rule used in the model. 
The retrograde signal diffuses from the synapses of the activated Kenyon cells by the first odor and the output neuron and affect the synapse weight between the activated Kenyon cells by the first odor and their inputs ( the activated projection neurons)if the amount of the received retrograde signal is high enough to trigger the change according to equation 4.7). The amount of the retrograde signal from each Kenyon cell's synapse depends on the current from the Kenyon cell's synapse inserted locally into the output neuron. As the amount of current into the output neuron induced by each spike depends on both spiking rate of the Kenyon cell and the synaptic weight between the Kenyon cell and the output neuron, the change of synapse weight between activated Kenyon cells by the first odor and the activated projection neurons is affected by both the first odor and shock pairing and consequently by an increase in the synaptic weight between the activated Kenyon cells and the projection neurons by the first odor (which increases the firing rate of Kenyon cells).

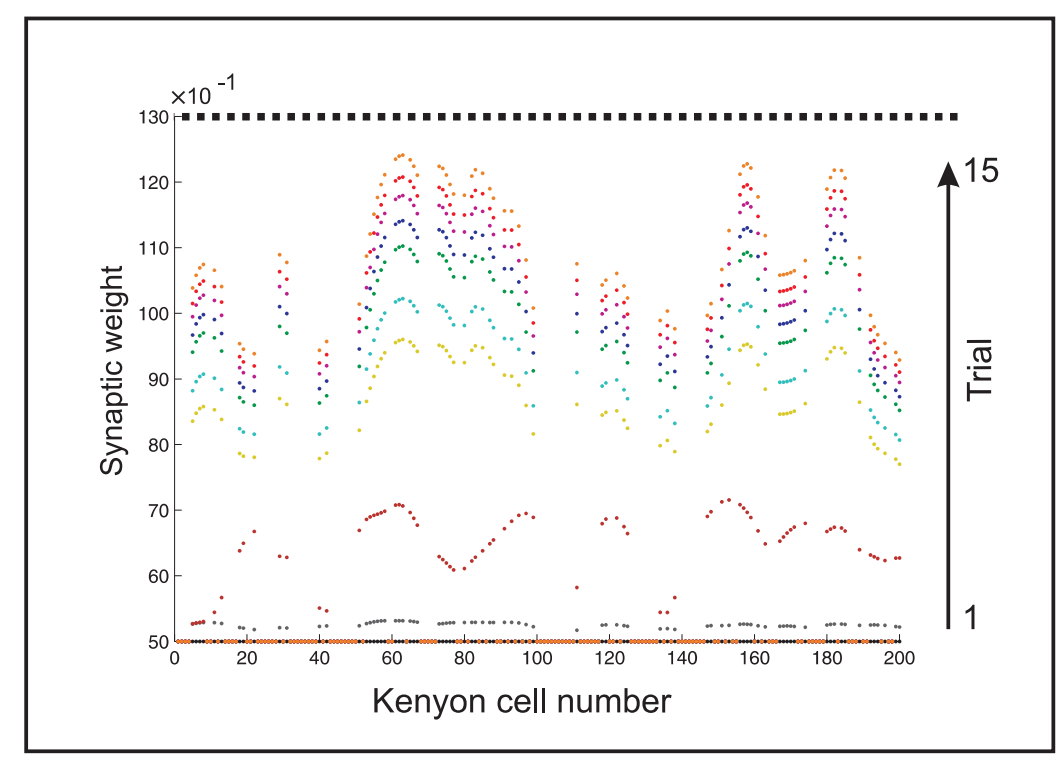

Figure 4.10: The change of synaptic weight between Kenyon cells and projection neurons for different trials of the first odor and shock pairing. The change of synapse weight of the activated Kenyon cells by the first odor is determined by the amount of received retrograde signal by each Kenyon cell. 
The change of synaptic weight between the activated neurons in the antennal lobe and the mushroom body and the mushroom body and the output neuron cause increase in the current from the activated Kenyon cells by the first odor into the output neuron which leads to the raise in the output neuron firing rate. A threshold equal 0.8 was used to model the emerging of avoidance behavior (Fig. 4.11). Limits for the maximum synaptic weight for both sites of learning are considered (dash line in Fig 4.9 and Fig. 4.10).

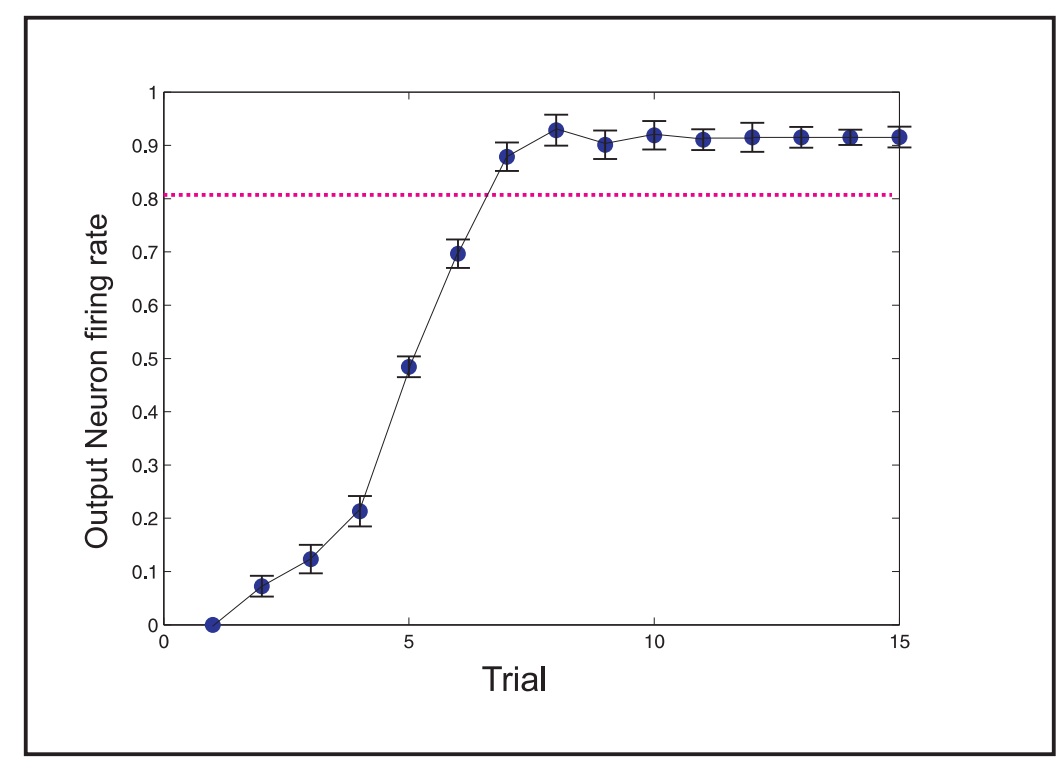

Figure 4.11: The change of output neuron's firing rate for different trials of the first odor and shock pairing. The pairing of the first odor and shock causes an increase in the synaptic weight between the first odor activated Kenyon cells and output neuron which consequently leads to increase in output neuron firing rate. A threshold equal to 0.8 was used to trigger the avoidance behavior. 


\subsubsection{Second phase}

In the second phase of the simulations, the second odor was paired with the first odor (trained odor). Although the second odor stimulated a set of the projection neurons that has no overlap with the activated projection neurons by the first odor, however, some Kenyon cells may be activated by both the first odor and the second odor. The activated Kenyon cells by both the first odor and the second odor are shown by black arrows in Fig. 4.12. These Kenyon cells produce high level of the retrograde signal. But at the beginning of the first odor and the second odor pairing, the activated Kenyon cells by the second odor stimulate very low level of the retrograde signal produced from the output neuron because of very low synaptic weight between the activated Kenyon cells by the second odor and the output neuron.

The change of the synaptic weight between the activated Kenyon cells by the second odor and the projection neurons are demonstrated in Fig. 4.13. The activated Kenyon cells by the first odor trigger the output neuron to release high amounts of the retrograde signal which diffuses into all Kenynon cells including those activated by the second odor. This increases the synaptic weight between the activated Kenyon cells by the second odor and the output neuron. At the end of pairing of the first and the second odors, high level of the retrograde signal are produced from output neuron induced by the second odor.

The change of synaptic weight between the activated Kenyon cells by the second odor and the output neuron at the end of the first odor and the second odor pairings, is shown in Fig. 4.14. Increase in firing rate of the activated Kenyon cells by the second odor, induced by high amounts of the diffused retrograde signal as well as high firing rate of the output neuron induced by the first odor presentation, leads to the increase in the synaptic weight between the activated Kenyon cells by the second odor and the output neuron according to the equations 4.8 and 4.9 . 


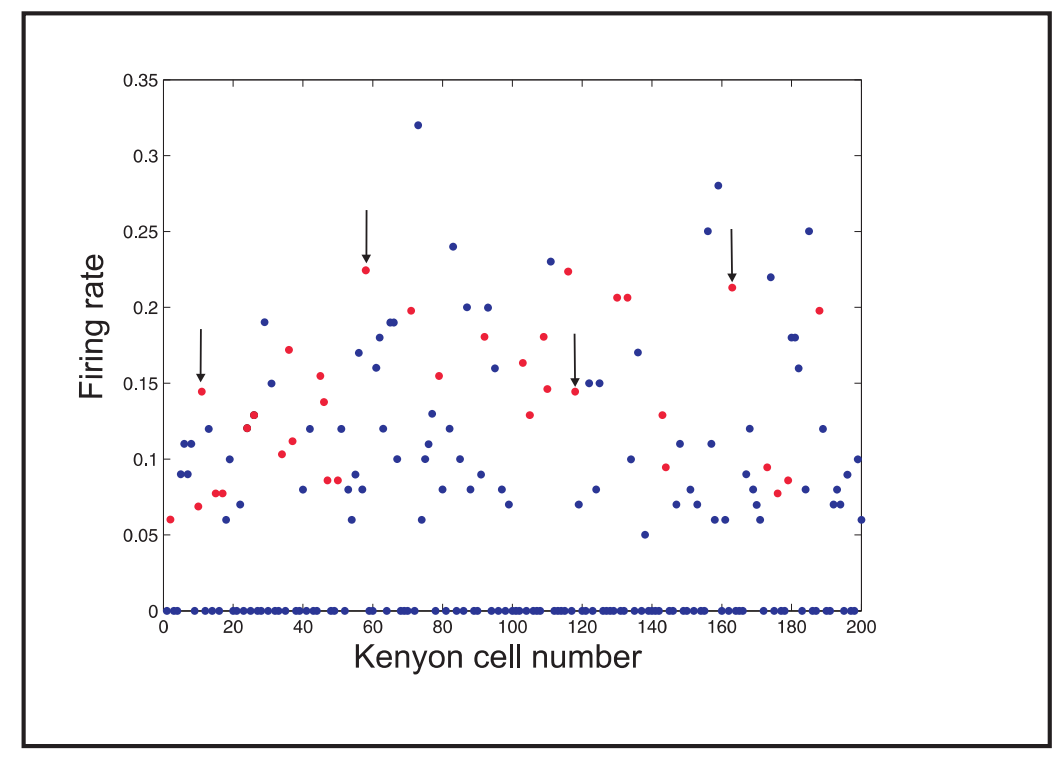

Figure 4.12: The firing rate of the activated Kenyon cells by the first and the second odor, blue and red circles, respectively. Although the first odor and the second odor stimulate separate set of the projection neurons, however, some Kenyon cells may be activated by both odors (shown by black arrows).

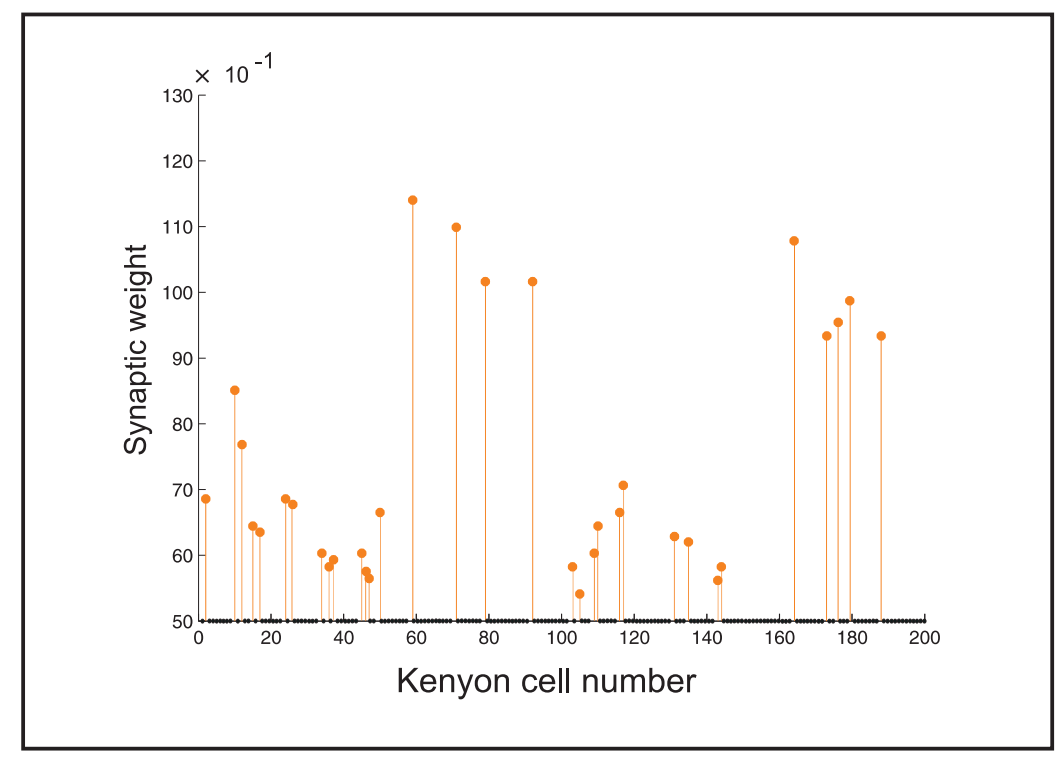

Figure 4.13: The synaptic weight between the activated Kenyon cells by the second odor and the projection neurons at the end of the first odor and the second odor pairings. The diffused retrograde signals triggered by the first odor presentation is received by the activated Kenyon cells by the second odor that causes an increase in the synaptic weight between the activated Kenyon cells by the second odor and the projection neurons. 


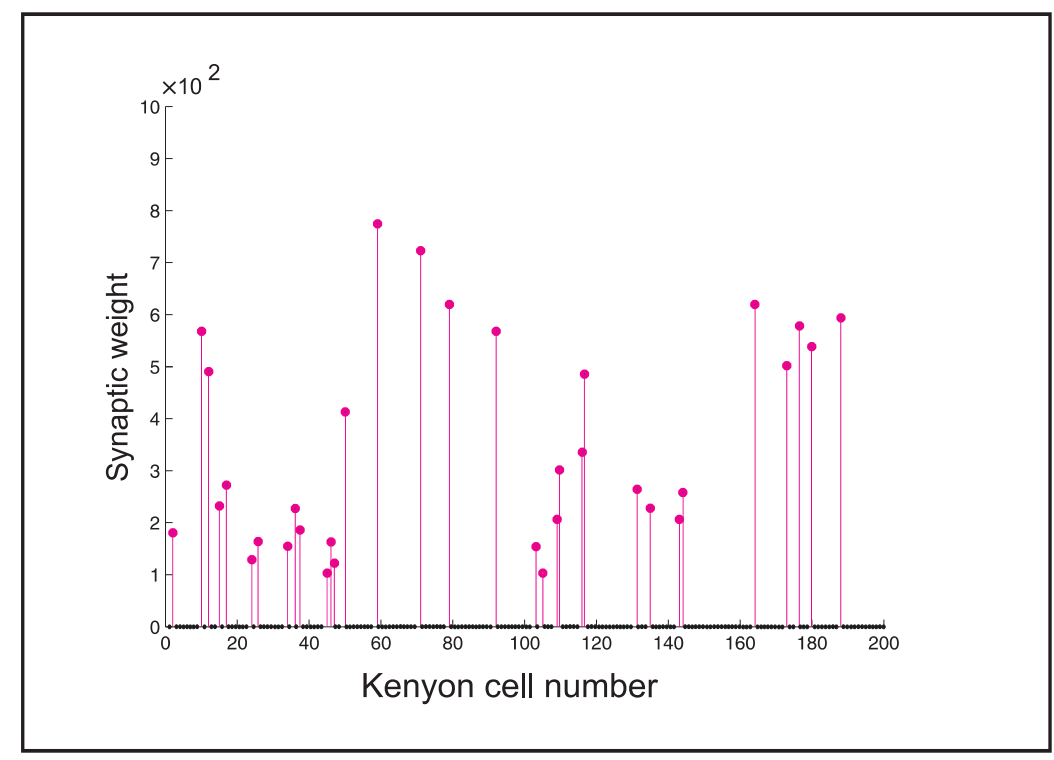

Figure 4.14: The synaptic weight of the activated Kenyon cells by the second odor and the output neuron at the end of the first odor and the second odor pairings. The change in synaptic weight between the activated Kenyon cells by the second odor and the projection neurons leads to an increase in firing rate of the Kenyon cells. This cause an increase in the synaptic weight between the activated Kenyon cells by the second odor and the output neuron according to Hebbian rule used in the model. 


\subsubsection{Third phase}

After the first odor and the second odor pairing, the second odor was presented to the insilico-fly individually to observe the effect of second phase. The firing rate of the output neuron for the second odor presenting is shown in Fig. 4.15. It shows that after the second phase, the second odor can elicit the output neuron to spike at high rate. It also illustrates that non-paired odor elicits no spiking in the output neuron.

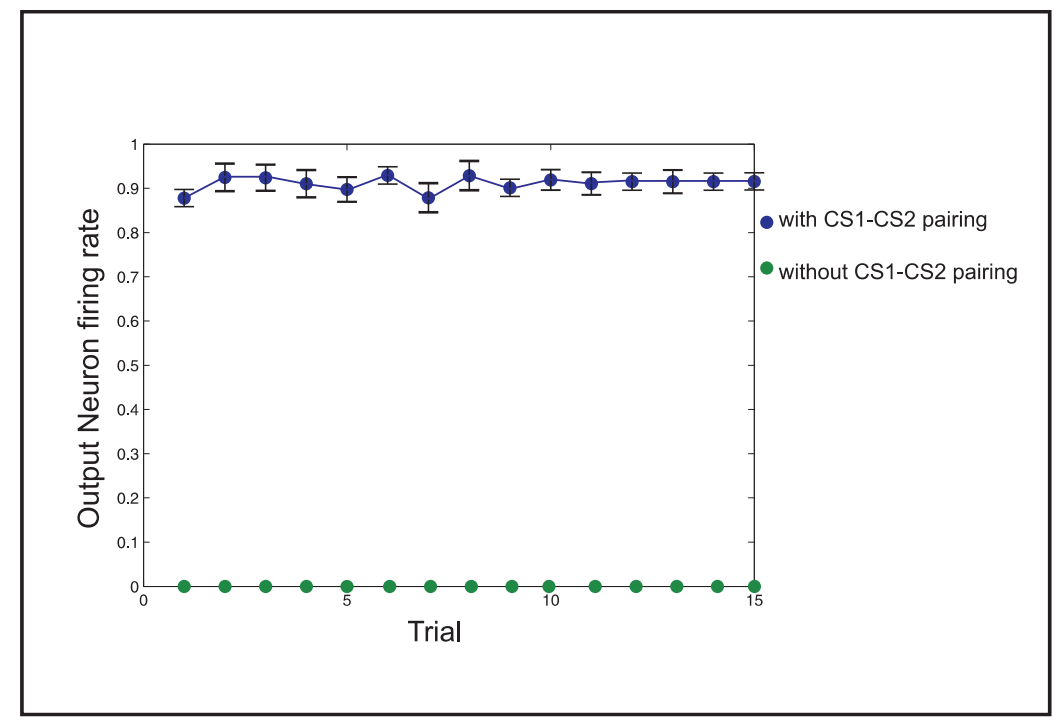

Figure 4.15: The output neuron firing rate stimulated by the second odor presenting after the first odor and the second odor pairing. The simulation shows that the second odor after pairing with the first odor can independently trigger avoidance behavior.

To test the role of the synaptic weight in these two learning place for retained learning, in a simulation, we set the synaptic weight between the antennal lobe and the mushroom body to its basal level (equal to 0.5). This leads to decrease in the output neuron's firing rate but it is gradually recovered into its original level after a few trials (Fig. 4.16 A).

Removing the synaptic weight between the mushroom body and the output neuron leads to permanent deactivation of the output neuron (Fig. $4.16 \mathrm{~A}$ ). It shows that gradually the synaptic weight gets its original values. In another simulation, the production of the retrograde signal from the output neuron to the mushroom body was blocked which resulted to the rapid decrease in the output neuron's firing rate (Fig. 4.16 B). 


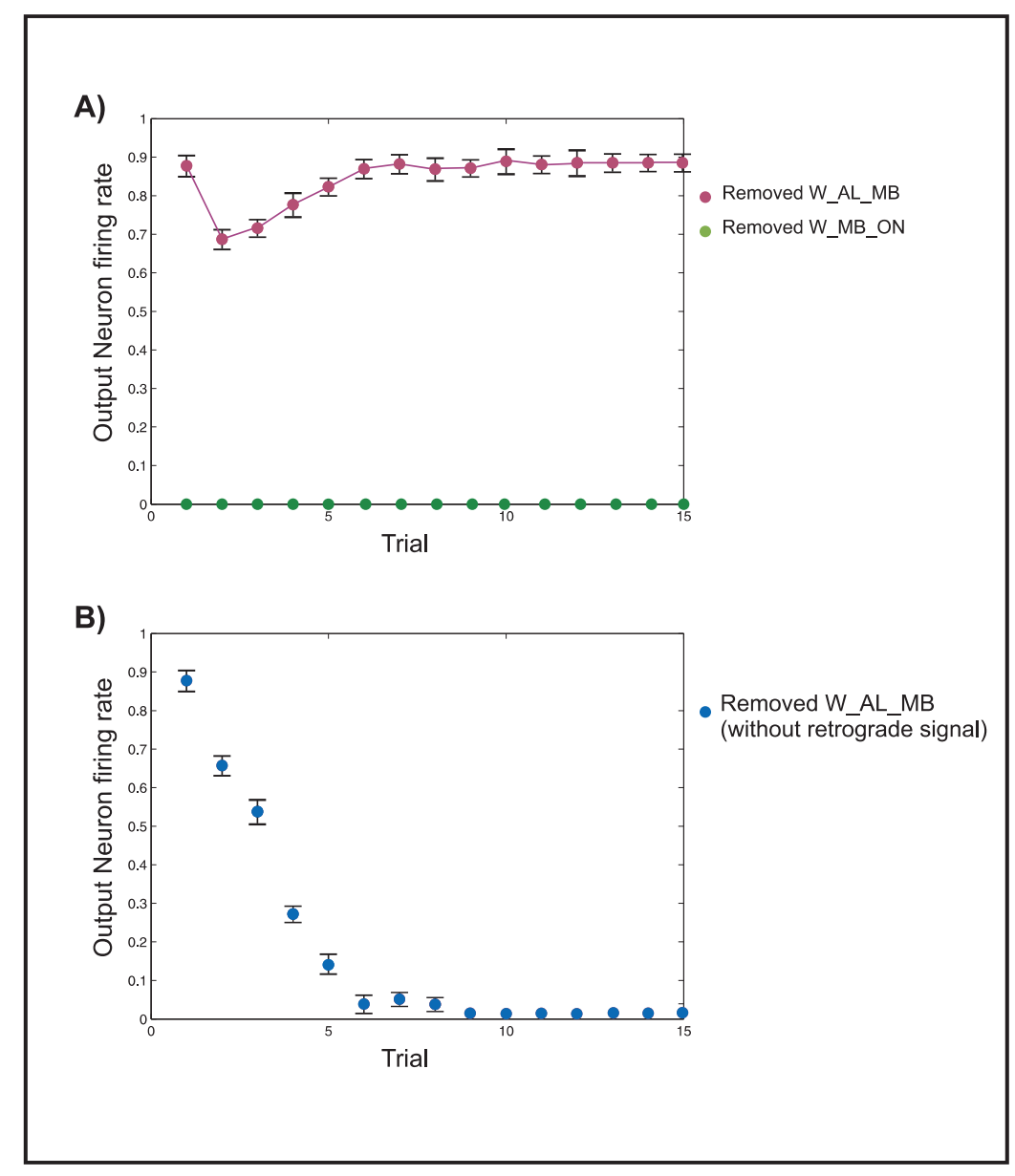

Figure 4.16: A) The effect of removing the synaptic weight between the projection neurons and the Kenyon cells, which are activated by the second odor. Removing the synaptic weight leads to decrease in the output neuron's firing rate that is raised gradually to high values after a few trials of the second odor presenting. It also shows that removing the updated synaptic weight between the mushroom body and the output neuron leads to deactivation of the output neuron. B) Removing the capability of diffusion of the retrograde signaling from the output neuron to the Kenyon cells leads to deactivation of the output neuron's spiking after a few trials. 
The recovery of synaptic efficacy between the activated Kenyon cells and the projection neurons by presenting the second odor is illustrated in Fig. 4.17. At the beginning, the synaptic weight between the activated Kenyon cells by the second odor and the output neuron is still high. It cause the release of high amounts of the retrograde signal which affect the synaptic weight of the activated Kenyon cells and the activated projection neurons by the second odor.

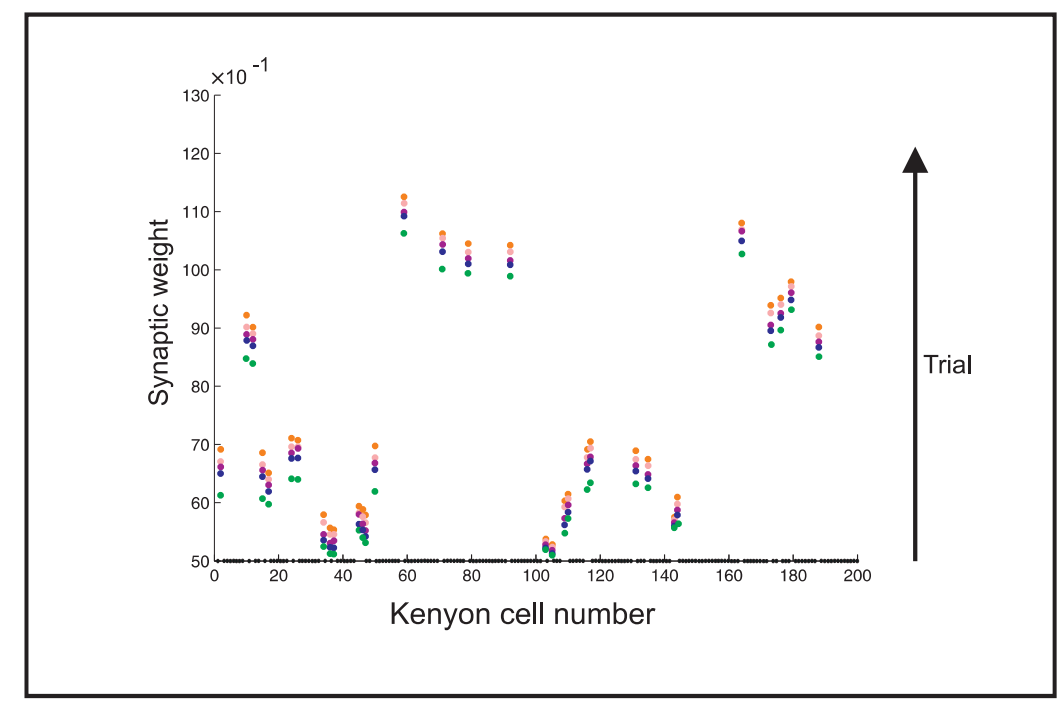

Figure 4.17: The change in the synaptic weight between the activated Kenyon cells by the second odor and the projection neurons after removing the updated synaptic weight through the first odor and the second odor pairings. The synaptic weight is increased again to high levels that is caused by diffusion of the retrograde signal from synapses between the Kenyon cells and the output neuron to synapses between the Kenyon cells and the projection neurons.

\subsubsection{Two-dimensional navigation}

To illustrate the process of first as well as second-order conditioning in the insilicofly developed in this model, a two- dimensional space was considered in which the fly navigates the environment. The result of training at the end of each phase of the simulations is shown in Fig. 4.18. It shows that at the end of the training, the second odor has produced the capability to be a predictor of the punishment. 


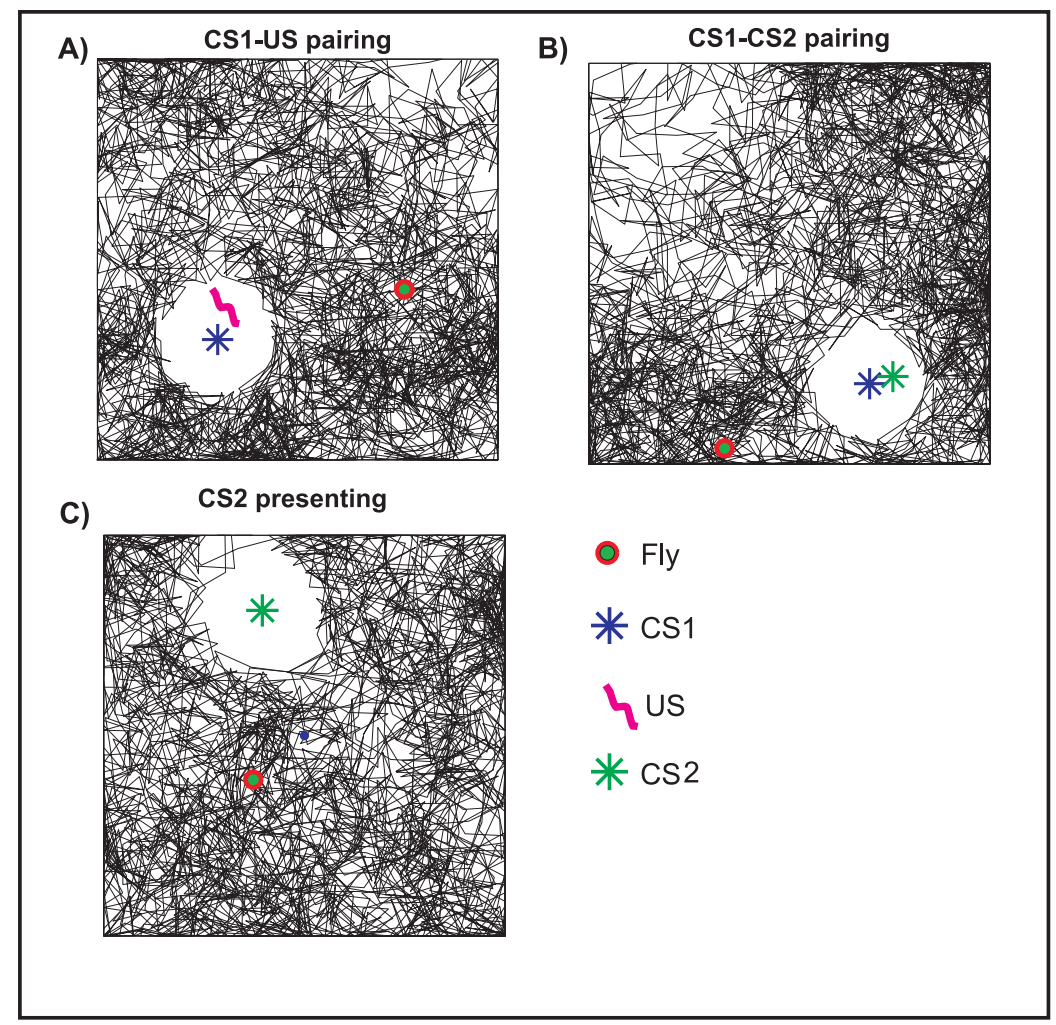

Figure 4.18: Two-dimensional simulation of the insilico-fly navigation through the conditioning process. The fly navigates the environment as random walk. When it is close enough to the food source, the fly may receive odor or both odor and the electric shock, depending on the protocol of each simulation. A) First order conditioning. The fly avoids the odor after trials of the first odor and shock pairings. B) The first and the second odor pairing. The fly immediately avoids the odors. C) The second odor presenting. Fly may avoid the second odor due to the frist and the second odors pairing phase. 


\subsection{Discussion}

Second order conditioning is one of the higher cognitive capabilities in animals . To understand the neural circuits underlying second order conditioning, neuroscientists have been studying insects' brain because of their partially well known structure and function. For this purpose, simulations and models help to develop new theories and design new testable predictions of Drosophila olfactory system.

Although an abstract model has been proposed to describe underlying mechanism of second order conditioning [Mizunami et al., 2009], no experiment has been conducted to test this theory in associative learning of the Drosophila olfactory system [Tabone and Belle, 2011].

The Insilico-fly model introduced in this study demonstrates the capability of producing of second order conditioning. On one hand, proposed model is based on experimental data about the anatomy and physiology of the Drosophila olfactory system as well as electrophysiological data on honeybees' brain. On the other hand, it is based on hypothetical mechanisms of non-synaptic communications of neurons which has been studied in vertebrate's neural systems. These neural communication needs to be experimentally investigated in insects' brains. In this modeling framework, it is assumed that learning takes place in two sites:

a) Synapses between the antennal lobe and the mushroom body and b) Synapses between the mushroom body and the output neuron.

The simulation of removing the updated synaptic weight between the activated Kenyon cells and the projection neurons which can be considered as short-term memory at the antennal lobe, may have some advantage to learn huge number of odors because different odors due to low number of neurons in the antennal lobe, may likely share activated sets of projection neurons while odors information is encoded in the mushroom body as sets of activated Kenyon cells (sparse coding) with very low degrees of overlap. The short-term memory in the antennal lobe, therefore, serves as a mechanism to prevent mistakes in specific association between odors and reward/punishment.

There are some challenges to propose this model as a neural circuit for second order conditioning in the Drosophila olfactory system: 1 . The assumption of the role of the output neuron's firing rate in eliciting of avoidance behavior: The role of output neuron in conditioning is still under research.

2. The retrograde signal production and diffusion from output neuron as a consequence of odor presentation: The role of non-synaptic communication of neurons has been studied in vertebrates and their role in health and diseases are well known. But these mechanisms need to be tested in insects' brain to investigate their role in their learning behaviors.

3. The role of change of synaptic efficacy between the activated Kenyon cells and the activated projection neurons in associative learning: The learning place in flies brain 
are not fully known. Both connections between the antennal lobe and the mushroom body and between the mushroom body and the output neuron are considered as the candidates places that may be involved in learning but they are still under research.

4. The Hebbian rule used in this model depends on the change of firing rate of Kenyon cells through the learning process. However, some studies have demonstrated that the Kenyon cells generate a few spikes in response to odor presentation [Turner et al., 2008], [Perez-Orive et al., 2002].

These observations are the main challenges to accept our model for second order conditioning in the Drosophila olfactory system.

However, to our knowledge, this is the first model to implement second order conditioning in artificial neural networks inspired by biological systems. Moreover, its simplicity in the structure and the neuron models (integrate an fire model) make it a powerful approach for some applications in artificial systems to develop new neural networks with capability to learn complicated tasks. 



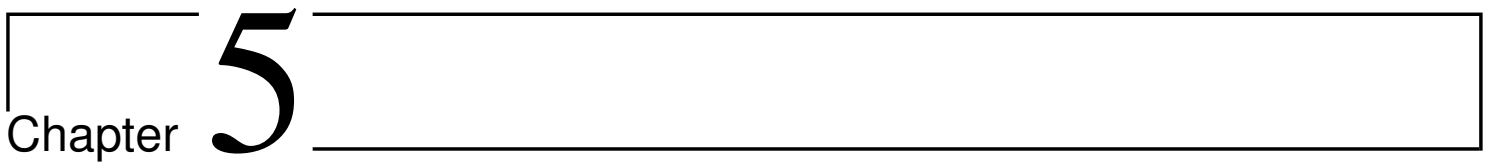

\section{Conclusion}

To understand the principles of information processing in animals' brains, the mechanisms of encoding different stimuli in the corresponding neural systems have to be analyzed. Some structural and physiological parameters are difficult to assess by experiments to study their role in different information processing tasks. For this purpose, models and simulations of neural processes play a significant role in determining the contribution of parameters in systems efficiency.

Information theory offers tools to measure the transferred information from the environment to brain areas where memory and learning takes place. Our information theoretic model of information processing in the Drosophila olfactory system provides a mathematical framework to measure the system efficiency with different parameter values. The model is not specific to Drosophila olfactory system and can be augmented to model other sensory systems with different architecture and physiology. The model assigns an optimal value for the activity of feedback inhibition in the Drosophila olfactory system and predicts thereby a pivotal role for inhibition to provide maximum information for example to optimally learn in associative learning processes.

One interesting physiological feature of the Kenyon cells is their 'sparse spiking' in response to odor presentation. Assuming that sparse spiking in the Kenyon cells is caused by high inhibition intensities, our method assigns very low system efficiency to the Drosophila olfactory system to encode odor information (Fig. 3.7). This illustrates that odor information (quality and concentration) may be mainly encoded by the sparseness of activated Kenyon cells in the mushroom body (number and location of the Kenyon cells responding to odor presentation).

Although information theory provides some measures of transferred information, simulations, however, seems to be good approaches to describe neural mechanisms of learning tasks at network level. Olfactory conditioning as an information processing mechanisms, has been studied by neuroscientists to understand the basic 


\section{Conclusion}

underlying mechanisms of complex brain functions. Although some roles of retrograde signaling in developing and normal functioning of neural systems have been found, however, the role of such neural communications in associative learning of Drosophila has not been fully studied yet [Smith et al., 2008].

Compared to other models for second order conditioning in insects

[Mizunami et al., 2009], our model is based on a different hypothesis of information processing during conditioning which proposes testable experiments to study the possible roles of non-synaptic communication in insects behavioral studies.

On the one hand, the proposed model for second order conditioning illustrates the capability of developing models based on integration of synaptic and non-synaptic communication to describe complex behaviors of animals. On the other hand, the simplicity of the used neuron model in the insilico-fly and the easy implementation of its mechanisms, make it an efficient method to implement learning tasks in artificial systems. 


\section{Bibliography}

[Abramson et al., 2010] Abramson, C. I., Giray, T., Mixson, T. A., Nolf, S. L., Wells, H., Kence, A., and Kence, M. (2010). Proboscis conditioning experiments with honeybees, Apis mellifera caucasica, with butyric acid and DEET mixture as conditioned and unconditioned stimuli. Journal of insect science, 10(122):122.

[Aceves-Piña and Quinn, 1979] Aceves-Piña, E. O. and Quinn, W. G. (1979). Learning in normal and mutant Drosophila larvae. Science, 206(4414):93-6.

[Arena et al., 2010] Arena, P., Berg, C., Patane, L., Strauss, R., and Termini, P. (2010). An insect brain computational model inspired by Drosophila melanogaster: Architecture description. The 2010 International Joint Conference on Neural Networks (IJCNN), pages 1-7.

[Assisi et al., 2007] Assisi, C., Stopfer, M., Laurent, G., and Bazhenov, M. (2007). Adaptive regulation of sparseness by feedforward inhibition. Nature neuroscience, 10(9):1176-84.

[Attneave, 1954] Attneave, F. (1954). Some informational aspects of visual perception. Psychological review, 61(3):183-93.

[Benton, 2008] Benton, R. (2008). Chemical sensing in Drosophila. Current opinion in neurobiology, 18(4):357-63.

[Benton et al., 2009] Benton, R., Vannice, K. S., Gomez-Diaz, C., and Vosshall, L. B. (2009). Variant ionotropic glutamate receptors as chemosensory receptors in Drosophila. Cell, 136(1):149-62.

[Bhandawat et al., 2007] Bhandawat, V., Olsen, S. R., Gouwens, N. W., Schlief, M. L., and Wilson, R. I. (2007). Sensory processing in the Drosophila antennal lobe increases reliability and separability of ensemble odor representations. Nature neuroscience, 10(11):1474-82. 


\section{Bibliography}

[Bicker, 1999] Bicker, G. (1999). Histochemistry of classical neurotransmitters in antennal lobes and mushroom bodies of the honeybee. Microscopy research and technique, 45(3):174-83.

[Bicker, 2001] Bicker, G. (2001). Nitric oxide: an unconventional messenger in the nervous system of an orthopteroid insect. Archives of insect biochemistry and physiology, 48(2):100-10.

[Borst and Theunissen, 1999] Borst, a. and Theunissen, F. E. (1999). Information theory and neural coding. Nature neuroscience, 2(11):947-57.

[Brennan and Keverne, 1997] Brennan, P. a. and Keverne, E. B. (1997). Neural mechanisms of mammalian olfactory learning. Progress in neurobiology, $51(4): 457-81$.

[Brody and Hopfield, 2003] Brody, C. D. and Hopfield, J. J. (2003). Simple networks for spike-timing-based computation, with application to olfactory processing. Neuron, 37(5):843-52.

[Broome et al., 2006] Broome, B. M., Jayaraman, V., and Laurent, G. (2006). Encoding and decoding of overlapping odor sequences. Neuron, 51(4):467-82.

[Busto et al., 2010] Busto, G. U., Cervantes-Sandoval, I., and Davis, R. L. (2010). Olfactory learning in Drosophila. Physiology, 25(6):338-46.

[Butcher et al., 2012] Butcher, N. J., Friedrich, A. B., Lu, Z., Tanimoto, H., and Meinertzhagen, I. a. (2012). Different classes of input and output neurons reveal new features in microglomeruli of the adult Drosophila mushroom body calyx. The Journal of comparative neurology, 520(10):2185-201.

[Caron et al., 2013] Caron, S. J. C., Ruta, V., Abbott, L. F., and Axel, R. (2013). Random convergence of olfactory inputs in the Drosophila mushroom body. $\mathrm{Na}$ ture, 497(7447):113-7.

[Cassenaer and Laurent, 2007] Cassenaer, S. and Laurent, G. (2007). Hebbian STDP in mushroom bodies facilitates the synchronous flow of olfactory information in locusts. Nature, 448(7154):709-13.

[Couto et al., 2005] Couto, A., Alenius, M., and Dickson, B. J. (2005). Molecular, anatomical, and functional organization of the Drosophila olfactory system. Current biology, 15(17):1535-47.

[Davis, 2005] Davis, R. L. (2005). Olfactory memory formation in Drosophila: from molecular to systems neuroscience. Annual review of neuroscience, 28:275-302.

[Debiec et al., 2006] Debiec, J., Doyère, V., Nader, K., and Ledoux, J. E. (2006). Directly reactivated, but not indirectly reactivated, memories undergo reconsolidation in the amygdala. PNAS, 103(9):3428-33. 
[Dimitrov et al., 2011] Dimitrov, A. G., Lazar, A. a., and Victor, J. D. (2011). Information theory in neuroscience. Journal of computational neuroscience, 30(1):1-5.

[Dubnau et al., 2001] Dubnau, J., Grady, L., Kitamoto, T., and Tully, T. (2001). Disruption of neurotransmission in Drosophila mushroom body blocks retrieval but not acquisition of memory. Nature, 411(6836):476-80.

[Farris, 2005] Farris, S. M. (2005). Evolution of insect mushroom bodies: old clues, new insights. Arthropod Structure \& Development, 34(3):211-234.

[Fiala, 2007] Fiala, A. (2007). Olfaction and olfactory learning in Drosophila: recent progress. Current opinion in neurobiology, 17(6):720-6.

[Finelli et al., 2008] Finelli, L. a., Haney, S., Bazhenov, M., Stopfer, M., and Sejnowski, T. J. (2008). Synaptic learning rules and sparse coding in a model sensory system. PLoS computational biology, 4(4):e1000062.

[Firestein, 2001] Firestein, S. (2001). How the olfactory system makes sense of scents. Nature, 413(6852):211-8.

[Fishilevich and Vosshall, 2005] Fishilevich, E. and Vosshall, L. B. (2005). Genetic and functional subdivision of the Drosophila antennal lobe. Current biology : $C B$, 15(17):1548-53.

[Gage et al., 2013] Gage, S. L., Daly, K. C., and Nighorn, A. (2013). Nitric oxide affects short-term olfactory memory in the antennal lobe of Manduca sexta. The Journal of experimental biology, 216(17):3294-300.

[García-Sanchez and Huerta, 2003] García-Sanchez, M. and Huerta, R. (2003). Design parameters of the fan-out phase of sensory systems. Journal of computational neuroscience, $15(1): 5-17$.

[Gerber and Stocker, 2007] Gerber, B. and Stocker, R. F. (2007). The Drosophila larva as a model for studying chemosensation and chemosensory learning: a review. Chemical senses, 32(1):65-89.

[Gewirtz, 2000] Gewirtz, J. C. (2000). Using Pavlovian Higher-Order Conditioning Paradigms to Investigate the Neural Substrates of Emotional Learning and Memory. Learning \& Memory, 7(5):257-266.

[Gu and Liljenström, 2007] Gu, Y. and Liljenström, H. (2007). Modelling efficiency in insect olfactory information processing. Bio Systems, 89(1-3):236-43.

[Guo et al., 1996] Guo, a., Li, L., Xia, S. Z., Feng, C. H., Wolf, R., and Heisenberg, M. (1996). Conditioned visual flight orientation in Drosophila: dependence on age, practice, and diet. Learning \& Memory, 3(1):49-59.

[Hallem and Carlson, 2006] Hallem, E. a. and Carlson, J. R. (2006). Coding of odors by a receptor repertoire. Cell, 125(1):143-60. 


\section{Bibliography}

[Heisenberg, 2003] Heisenberg, M. (2003). Mushroom body memoir: from maps to models. Nature Reviews Neuroscience, 4(4):266-75.

[Heisenberg et al., 1985] Heisenberg, M., Borst, a., Wagner, S., and Byers, D. (1985). Drosophila mushroom body mutants are deficient in olfactory learning. Journal of neurogenetics, 2(1):1-30.

[Honegger et al., 2011] Honegger, K. S., Campbell, R. a. a., and Turner, G. C. (2011). Cellular-resolution population imaging reveals robust sparse coding in the Drosophila mushroom body. The Journal of neuroscience : the official journal of the Society for Neuroscience, 31(33):11772-85.

[Huerta and Nowotny, 2009] Huerta, R. and Nowotny, T. (2009). Fast and robust learning by reinforcement signals: explorations in the insect brain. Neural computation, 21(8):2123-51.

[Huerta et al., 2004] Huerta, R., Nowotny, T., García-Sanchez, M., Abarbanel, H. D. I., and Rabinovich, M. I. (2004). Learning classification in the olfactory system of insects. Neural computation, 16(8):1601-40.

[Hussaini et al., 2007a] Hussaini, S. A., Komischke, B., Menzel, R., and Lachnit, H. (2007a). Forward and backward second-order Pavlovian conditioning in honeybees. pages $678-683$.

[Hussaini et al., 2007b] Hussaini, S. A., Komischke, B., Menzel, R., and Lachnit, H. (2007b). Forward and backward second-order Pavlovian conditioning in honeybees. pages $678-683$.

[Izhikevich, 2007] Izhikevich, E. M. (2007). Solving the distal reward problem through linkage of STDP and dopamine signaling. Cerebral cortex, 17(10):244352.

[Jara et al., 2006] Jara, E., Vila, J., and Maldonado, A. (2006). Second-order conditioning of human causal learning. Learning and Motivation, 37(3):230-246.

[Jefferis et al., 2007] Jefferis, G. S. X. E., Potter, C. J., Chan, A. M., Marin, E. C., Rohlfing, T., Maurer, C. R., and Luo, L. (2007). Comprehensive maps of Drosophila higher olfactory centers: spatially segregated fruit and pheromone representation. Cell, 128(6):1187-203.

[Jortner et al., 2007] Jortner, R. a., Farivar, S. S., and Laurent, G. (2007). A simple connectivity scheme for sparse coding in an olfactory system. The Journal of neuroscience, 27(7):1659-69.

[Karazinov and Boakes, 2007] Karazinov, D. M. and Boakes, R. a. (2007). Secondorder conditioning in human predictive judgements when there is little time to think. Quarterly journal of experimental psychology (2006), 60(3):448-60. 
[Kay and Stopfer, 2006] Kay, L. M. and Stopfer, M. (2006). Information processing in the olfactory systems of insects and vertebrates. Seminars in cell $\mathcal{E}$ developmental biology, 17(4):433-42.

[Keene and Waddell, 2007] Keene, A. C. and Waddell, S. (2007). Drosophila olfactory memory: single genes to complex neural circuits. Nature reviews. Neuroscience, 8(5):341-54.

[Khurana et al., 2009] Khurana, S., Abu Baker, M. B., and Siddiqi, O. (2009). Odour avoidance learning in the larva of Drosophila melanogaster. Journal of biosciences, 34(4):621-31.

[Kivity et al., 2009] Kivity, S., Ortega-Hernandez, O. D., and Shoenfeld, Y. (2009). Olfaction-a window to the mind. The Israel Medical Association journal : IMAJ, 11(4):238-43.

[Krashes et al., 2007] Krashes, M. J., Keene, A. C., Leung, B., Armstrong, J. D., and Waddell, S. (2007). Sequential use of mushroom body neuron subsets during drosophila odor memory processing. Neuron, 53(1):103-15.

[Kwon, 2004] Kwon, H.-W. (2004). Spatial learning in the restrained American cockroach Periplaneta americana. Journal of Experimental Biology, 207(2):377383 .

[Laissue et al., 1999] Laissue, P. P., Reiter, C., Hiesinger, P. R., Halter, S., Fischbach, K. F., and Stocker, R. F. (1999). Three-dimensional reconstruction of the antennal lobe in Drosophila melanogaster. The Journal of comparative neurology, 405(4):543-52.

[Laloi et al., 1999] Laloi, D., Sandoz, J. C., Marchesi, A., Pouvreau, A., Tas, J. N., and Poppy, G. (1999). Olfactory conditioning of the proboscis extension in bumble bees. (1995):123-129.

[Laurent, 1999] Laurent, G. (1999). A Systems Perspective on Early Olfactory Coding. Science, 286(5440):723-728.

[Laurent et al., 2001a] Laurent, G., Stopfer, M., Friedrich, R. W., Rabinovich, M. I., Volkovskii, a., and Abarbanel, H. D. (2001a). Odor encoding as an active, dynamical process: experiments, computation, and theory. Annual review of neuroscience, 24:263-97.

[Laurent et al., 2001b] Laurent, G., Stopfer, M., Friedrich, R. W., Rabinovich, M. I., Volkovskii, A., and Abarbanel, H. D. I. (2001b). Odor encoding as an active, dynamical process : Experiments , Computation ,.

[Lee et al., 2003] Lee, D., Su, H., and Dowd, D. K. O. (2003). Inhibitory Synaptic Transmission in Drosophila Neurons. 23(11):4625-4634. 


\section{Bibliography}

[Leiss et al., 2009] Leiss, F., Groh, C., Butcher, N. J., Meinertzhagen, I. a., and Tavosanis, G. (2009). Synaptic organization in the adult Drosophila mushroom body calyx. The Journal of comparative neurology, 517(6):808-24.

[Lin et al., 2014] Lin, A. C., Bygrave, A. M., de Calignon, A., Lee, T., and Miesenböck, G. (2014). Sparse, decorrelated odor coding in the mushroom body enhances learned odor discrimination. Nature Neuroscience, pages 1-12.

[Liu et al., 2009] Liu, X., Buchanan, M. E., Han, K.-A., and Davis, R. L. (2009). The GABAA receptor RDL suppresses the conditioned stimulus pathway for olfactory learning. The Journal of neuroscience : the official journal of the Society for Neuroscience, 29(5):1573-9.

[Liu and Davis, 2009] Liu, X. and Davis, R. L. (2009). The GABAergic anterior paired lateral neuron suppresses and is suppressed by olfactory learning. Nature neuroscience, 12(1):53-9.

[Liu et al., 2007] Liu, X., Krause, W. C., and Davis, R. L. (2007). GABAA receptor RDL inhibits Drosophila olfactory associative learning. Neuron, 56(6):1090-102.

[Luo and Zhu, 2011] Luo, C.-X. and Zhu, D.-Y. (2011). Research progress on neurobiology of neuronal nitric oxide synthase. Neuroscience bulletin, 27(1):23-35.

[Malnic et al., 1999] Malnic, B., Hirono, J., Sato, T., Buck, L. B., and Hughes, H. (1999). Combinatorial Receptor Codes for Odors. 96:713-723.

[Marin et al., 2002] Marin, E. C., Jefferis, G. S. X. E., Komiyama, T., Zhu, H., and Luo, L. (2002). Representation of the glomerular olfactory map in the Drosophila brain. Cell, 109(2):243-55.

[Masek and Heisenberg, 2008] Masek, P. and Heisenberg, M. (2008). Distinct memories of odor intensity and quality in Drosophila. Proceedings of the National Academy of Sciences of the United States of America, 105(41):15985-90.

[Mcguire et al., 2005] Mcguire, S. E., Deshazer, M., and Davis, R. L. (2005). Thirty years of olfactory learning and memory research in Drosophila melanogaster. $76: 328-347$.

[McGuire et al., 2003] McGuire, S. E., Le, P. T., Osborn, A. J., Matsumoto, K., and Davis, R. L. (2003). Spatiotemporal rescue of memory dysfunction in Drosophila. Science, 302(5651):1765-8.

[Menzel and Manz, 2005] Menzel, R. and Manz, G. (2005). Neural plasticity of mushroom body-extrinsic neurons in the honeybee brain. The Journal of experimental biology, 208(Pt 22):4317-32.

[Miller et al., 1995] Miller, R. R., Barnet, R. C., and Grahame, N. J. (1995). Assessment of the Rescorla-Wagner model. Psychological bulletin, 117(3):363-86. 
[Milner et al., 1998] Milner, B., Squire, L. R., and Kandel, E. R. (1998). and the Study of Memory. 20:445-453.

[Mizunami et al., 2009] Mizunami, M., Unoki, S., Mori, Y., Hirashima, D., Hatano, A., and Matsumoto, Y. (2009). Roles of octopaminergic and dopaminergic neurons in appetitive and aversive memory recall in an insect. BMC biology, 7:46.

[Mombaerts, 2001] Mombaerts, P. (2001). How smell develops. Nature Neuroscience, 4(november):1192-1198.

[Ng et al., 2002] Ng, M., Roorda, R. D., Lima, S. Q., Zemelman, B. V., Morcillo, P., and Miesenböck, G. (2002). Transmission of olfactory information between three populations of neurons in the antennal lobe of the fly. Neuron, 36(3):463-74.

[Olsen et al., 2007] Olsen, S. R., Bhandawat, V., and Wilson, R. I. (2007). Excitatory interactions between olfactory processing channels in the Drosophila antennal lobe. Neuron, 54(1):89-103.

[Olsen and Wilson, 2008] Olsen, S. R. and Wilson, R. I. (2008). Cracking neural circuits in a tiny brain: new approaches for understanding the neural circuitry of Drosophila. Trends in neurosciences, 31(10):512-20.

[Panzeri et al., 2007] Panzeri, S., Senatore, R., Montemurro, M. a., and Petersen, R. S. (2007). Correcting for the sampling bias problem in spike train information measures. Journal of neurophysiology, 98(3):1064-72.

[Papadopoulou et al., 2011] Papadopoulou, M., Cassenaer, S., Nowotny, T., and Laurent, G. (2011). Normalization for sparse encoding of odors by a wide-field interneuron. Science, 332(6030):721-5.

[Pauls et al., 2010] Pauls, D., Pfitzenmaier, J. E. R., Krebs-Wheaton, R., Selcho, M., Stocker, R. F., and Thum, A. S. (2010). Electric shock-induced associative olfactory learning in Drosophila larvae. Chemical senses, 35(4):335-46.

[Perez-Orive et al., 2002] Perez-Orive, J., Mazor, O., Turner, G. C., Cassenaer, S., Wilson, R. I., and Laurent, G. (2002). Oscillations and sparsening of odor representations in the mushroom body. Science, 297(5580):359-65.

[Python and Stocker, 2002] Python, F. and Stocker, R. F. (2002). Immunoreactivity against choline acetyltransferase, gamma-aminobutyric acid, histamine, octopamine, and serotonin in the larval chemosensory system of Dosophila melanogaster. The Journal of comparative neurology, 453(2):157-67.

[Rahotte et al., 1977] Rahotte, M. E., Griffin, R. W., and Cheryl, L. (1977). Secondorder conditioning of the pigeon 's keypeck. Animal Learning $\&$ Behavior, 5(I):2538.

[Regehr et al., 2009] Regehr, W. G., Carey, M. R., and Best, A. R. (2009). Activitydependent regulation of synapses by retrograde messengers. Neuron, 63(2):154-70. 


\section{Bibliography}

[Ren et al., 2012] Ren, Q., Li, H., Wu, Y., Ren, J., and Guo, A. (2012). A GABAergic inhibitory neural circuit regulates visual reversal learning in Drosophila. The Journal of neuroscience, 32(34):11524-38.

[Riemensperger et al., 2005] Riemensperger, T., Völler, T., Stock, P., Buchner, E., and Fiala, A. (2005). Punishment prediction by dopaminergic neurons in Drosophila. Current biology, 15(21):1953-60.

[Satoh et al., 2010] Satoh, R., Oizumi, M., Kazama, H., and Okada, M. (2010). Mechanisms of maximum information preservation in the Drosophila antennal lobe. PloS one, 5(5):e10644.

[Schlief and Wilson, 2007] Schlief, M. L. and Wilson, R. I. (2007). Olfactory processing and behavior downstream from highly selective receptor neurons. Nature neuroscience, 10(5):623-30.

[Schwaerzel et al., 2003] Schwaerzel, M., Monastirioti, M., Scholz, H., Friggi-Grelin, F., Birman, S., and Heisenberg, M. (2003). Dopamine and octopamine differentiate between aversive and appetitive olfactory memories in Drosophila. The Journal of neuroscience, 23(33):10495-502.

[Séjourné et al., 2011] Séjourné, J., Plaçais, P.-y., Aso, Y., Siwanowicz, I., Trannoy, S., Thoma, V., Tedjakumala, S. R., Rubin, G. M., Tchénio, P., Ito, K., Isabel, G., Tanimoto, H., and Preat, T. (2011). Mushroom body efferent neurons responsible for aversive olfactory memory retrieval in Drosophila. Nature Publishing Group, 14(7):903-910.

[Selcho et al., 2009] Selcho, M., Pauls, D., Han, K.-A., Stocker, R. F., and Thum, A. S. (2009). The role of dopamine in Drosophila larval classical olfactory conditioning. PloS one, 4(6):e5897.

[Shang et al., 2007] Shang, Y., Claridge-Chang, A., Sjulson, L., Pypaert, M., and Miesenböck, G. (2007). Excitatory local circuits and their implications for olfactory processing in the fly antennal lobe. Cell, 128(3):601-12.

[Shlens et al., 2009] Shlens, J., Field, G. D., Gauthier, J. L., Greschner, M., Litke, A. M., and Chichilnisky, E. J. (2009). NIH Public Access. Journal of Neuroscience, 29(15):5022-5031.

[Silbering et al., 2008] Silbering, A. F., Okada, R., Ito, K., and Galizia, C. G. (2008). Olfactory information processing in the Drosophila antennal lobe: anything goes? The Journal of neuroscience, 28(49):13075-87.

[Sinakevitch et al., 2001] Sinakevitch, I., Farris, S. M., and Strausfeld, N. J. (2001). Immunoreactivity Identifies Chemically Distinct Subdivisions of Kenyon Cells in the Cockroach Mushroom Body. The Journal of Comparative Neurobiology, 367(439):352-367. 
[Singer et al., 2009] Singer, J. H., Glowatzki, E., Moser, T., Strowbridge, B. W., Bhandawat, V., and Sampath, A. P. (2009). Functional properties of synaptic transmission in primary sense organs. The Journal of neuroscience, 29(41):128026 .

[Singh and Lesica, 2010] Singh, A. and Lesica, N. a. (2010). Incremental mutual information: a new method for characterizing the strength and dynamics of connections in neuronal circuits. PLoS computational biology, 6(12):e1001035.

[Smith et al., 2008] Smith, D., Wessnitzer, J., and Webb, B. (2008). A model of associative learning in the mushroom body. Biological cybernetics, 99(2):89-103.

[Steinert et al., 2010] Steinert, J. R., Chernova, T., and Forsythe, I. D. (2010). Nitric oxide signaling in brain function, dysfunction, and dementia. The Neuroscientist : a review journal bringing neurobiology, neurology and psychiatry, 16(4):435-52.

[Stocker, 1994] Stocker, R. F. (1994). Cell \& Tissue Review article The organization of the chemosensory system in Drosophila melanogaster : a rewiew. Cell Tissue Research, 275:3-26.

[Stocker et al., 1997] Stocker, R. F., Heimbeck, G., Gendre, N., and de Belle, J. S. (1997). Neuroblast ablation in Drosophila P[GAL4] lines reveals origins of olfactory interneurons. Journal of neurobiology, 32(5):443-56.

[Stocker et al., 1990] Stocker, R. F., Lienhard, M. C., Borst, A., and Fischbach, K. (1990). Researdl in Drosophila melanogaster. Cell Tissue Research, 262:9-34.

[Stopfer et al., 2003] Stopfer, M., Jayaraman, V., and Laurent, G. (2003). Intensity versus identity coding in an olfactory system. Neuron, 39(6):991-1004.

[Strausfeld and Hildebrand, 1999] Strausfeld, N. J. and Hildebrand, J. G. (1999). Olfactory systems: common design, uncommon origins? Current opinion in neurobiology, 9(5):634-9.

[Strausfeld et al., 2003] Strausfeld, N. J., Sinakevitch, I., and Vilinsky, I. (2003). The mushroom bodies of Drosophila melanogaster: an immunocytological and golgi study of Kenyon cell organization in the calyces and lobes. Microscopy research and technique, 62(2):151-69.

[Szyszka et al., 2005] Szyszka, P., Ditzen, M., Galkin, A., Galizia, C. G., Menzel, R., Giovanni, C., and Sparsening, R. M. (2005). Sparsening and Temporal Sharpening of Olfactory Representations in the Honeybee Mushroom Bodies. Journal of Neurophysiology, 94:3303-3313.

[Tabone and Belle, 2011] Tabone, C. J. and Belle, J. S. D. (2011). Second-order conditioning in Drosophila. pages 250-253. 


\section{Bibliography}

[Tanaka et al., 2008] Tanaka, N. K., Tanimoto, H., and Ito, K. (2008). Neuronal assemblies of the Drosophila mushroom body. The Journal of comparative neurology, 508(5):711-55.

[Tao and Poo, 2001] Tao, H. W. and Poo, M. (2001). Retrograde signaling at central synapses. PNAS, 98(20):11009-15.

[Tempel et al., 1983] Tempel, B. L., Bonini, N., Dawson, D. R., and Quinn, W. G. (1983). Reward learning in normal and mutant Drosophila. PNAS, 80(5):1482-6.

[Triesch, 2007] Triesch, J. (2007). Synergies between intrinsic and synaptic plasticity mechanisms. Neural computation, 19(4):885-909.

[Tully and Quinn, 1985] Tully, T. and Quinn, W. G. (1985). Classical conditioning and retention in normal and mutant Drosophila melanogaster. Journal of comparative physiology. A, Sensory, neural, and behavioral physiology, 157(2):263-77.

[Turner et al., 2008] Turner, G. C., Bazhenov, M., and Laurent, G. (2008). Olfactory representations by Drosophila mushroom body neurons. Journal of neurophysiology, 99(2):734-46.

[Turrigiano, 2011] Turrigiano, G. (2011). Too many cooks? Intrinsic and synaptic homeostatic mechanisms in cortical circuit refinement. Annual review of neuroscience, 34:89-103.

[Vanni and Rosenström, 2011] Vanni, S. and Rosenström, T. (2011). Local nonlinear interactions in the visual cortex may reflect global decorrelation. Journal of computational neuroscience, 30(1):109-24.

[Vosshall, 2000] Vosshall, L. B. (2000). Olfaction in Drosophila. Current opinion in neurobiology, 10(4):498-503.

[Vosshall et al., 1999] Vosshall, L. B., Amrein, H., Morozov, P. S., Rzhetsky, a., and Axel, R. (1999). A spatial map of olfactory receptor expression in the Drosophila antenna. Cell, 96(5):725-36.

[Vosshall et al., 2000] Vosshall, L. B., Wong, a. M., and Axel, R. (2000). An olfactory sensory map in the fly brain. Cell, 102(2):147-59.

[Wall and Glackin, 2013] Wall, J. and Glackin, C. (2013). Spiking neural network connectivity and its potential for temporal sensory processing and variable binding. Frontiers in computational neuroscience, 7:182.

[Wang et al., 2004] Wang, Y., Guo, H.-F., Pologruto, T. a., Hannan, F., Hakker, I., Svoboda, K., and Zhong, Y. (2004). Stereotyped odor-evoked activity in the mushroom body of Drosophila revealed by green fluorescent protein-based Ca2+ imaging. The Journal of neuroscience, 24(29):6507-14. 
[Wilson and Laurent, 2005] Wilson, R. I. and Laurent, G. (2005). Role of GABAergic inhibition in shaping odor-evoked spatiotemporal patterns in the Drosophila antennal lobe. The Journal of neuroscience, 25(40):9069-79.

[Wilson et al., 2004] Wilson, R. I., Turner, G. C., and Laurent, G. (2004). Transformation of olfactory representations in the Drosophila antennal lobe. Science, 303(5656):366-70.

[Wong et al., 2002] Wong, A. M., Wang, J. W., and Axel, R. (2002). Spatial representation of the glomerular map in the Drosophila protocerebrum. Cell, $109(2): 229-41$.

[Wüstenberg et al., 2004] Wüstenberg, D. G., Boytcheva, M., Grünewald, B., Byrne, J. H., Menzel, R., and Baxter, D. a. (2004). Current- and voltage-clamp recordings and computer simulations of Kenyon cells in the honeybee. Journal of neurophysiology, 92(4):2589-603.

[Yan et al., 2012] Yan, X.-B., Ouyang, W., Li, G., and Duan, K.-M. (2012). Involvement of neuronal nitric oxide synthase in cognitive impairment in isofluranetreated rats. Neuroscience letters, 506(2):240-4.

[Yao et al., 2005] Yao, C. A., Ignell, R., and Carlson, J. R. (2005). Chemosensory coding by neurons in the coeloconic sensilla of the Drosophila antenna. The Journal of neuroscience, 25(37):8359-67.

[Yarali et al., 2009] Yarali, A., Ehser, S., Hapil, F. Z., Huang, J., and Gerber, B. (2009). Odour intensity learning in fruit flies. Proceedings. Biological sciences / The Royal Society, 276(1672):3413-20.

[Yu et al., 2004] Yu, D., Ponomarev, A., and Davis, R. L. (2004). Altered representation of the spatial code for odors after olfactory classical conditioning; memory trace formation by synaptic recruitment. Neuron, 42(3):437-49.

[Yusuyama and Meinertzhagen, 2002] Yusuyama, K. and Meinertzhagen, I. A. N. A. (2002). Synaptic Organization of the Mushroom Body Calyx in Drosophila melanogaster. Journal of Comparative physiology, 226:211-226.

[Zars, 2000] Zars, T. (2000). Localization of a Short-Term Memory in Drosophila. Science, 288(5466):672-675. 



\section{List of Figures}

1.1 Schematic illustration of the Drosophila olfactory system . . . . . . . 11

2.1 Schematic illustration of the information theoretic model of the Drosophila olfactory system . . . . . . . . . . . . . . . . 20 20

2.2 The second-order conditioning behavioral paradigms . . . . . . . . . 21

2.3 The structure of the proposed model for second-order conditioning . . 21

3.1 Schematic illustration of the model structure in information theoretic approach . . . . . . . . . . . . . . . . . 24

3.2 The relation between average Kenyon cells activity and probability of feedback inhibition for different parameter values . . . . . . . . . 26

3.3 Mutual information between the environment (here 5 odors) and Kenyon cells for different firing thresholds of Kenyon cells and connectivity rate between the antennal lobe and the mushroom body . . 30

3.4 Mutual information between the environment and 10 Kenyon cells or 20 Kenyon cells for different thresholds . . . . . . . . . . . . . . 31

3.5 Mutual information between the environment ( 5 odors) and 10 Kenyon cells with the effect of inhibition on Kenyon cells and high odor concentration . . . . . . . . . . . . . . . . 32

3.6 Mutual information between the mushroom body and the environ-

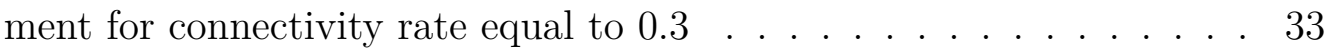

3.7 Mutual information between mushroom body and the environment . . 33

4.1 The cellular mechanism of affecting the synaptic efficacy by nitric oxide 39

4.2 The retrograde signaling hypothesis . . . . . . . . . . . . . . 39

4.3 The simulation of the dopaminergic neurons' activity in response to a second electric shock . . . . . . . . . . . . . . . . . 43

4.4 Production of the retrograde signal . . . . . . . . . . . . . . 45

4.5 The neural events underlying the first phase of the simulations . . . . 48

4.6 The neural events underlying the second phase of the simulations . . 50

4.7 The neural events underlying the third phase of simulations . . . . . 51 
4.8 The amount of the received retrograde signal by each synapse depends on its distance from the production sites of the retrograde signal . . . 53

4.9 The change of synaptic weight between the Kenyon cells and the output neuron for different trials of the first odor and shock pairing . 54

4.10 The change of synaptic weight between Kenyon cells and projection neurons for different trials of the first odor and shock pairing . . . . . 55

4.11 The change of output neuron's firing rate for different trials of the first odor and shock pairing . . . . . . . . . . . . . 56

4.12 The firing rate of the activated Kenyon cells by the first and the second odor . . . . . . . . . . . . . . . . . . . . 58

4.13 The synaptic weight between the activated Kenyon cells by the second odor and the projection neurons at the end of the first odor and the second odor pairings . . . . . . . . . . . . . . . 5 58

4.14 The synaptic weight of the activated Kenyon cells by the second odor and the output neuron at the end of the first odor and the second odor pairings . . . . . . . . . . . . . . . . . . 59

4.15 The output neuron firing rate stimulated by the second odor presenting after the first odor and the second odor pairing . . . . . . . . 60

4.16 The effect of removing the synaptic weight between the projection neurons and the Kenyon cells . . . . . . . . . . . . . . . 61

4.17 The change in the synaptic weight between the activated Kenyon cells by the second odor and the projection neurons after removing the updated synaptic weight through the first odor and the second odor pairings . . . . . . . . . . . . . . . . . . 62

4.18 Two-dimensional simulation of the insilico-fly navigation through the conditioning process . . . . . . . . . . . . . 6 63 


\section{List of Tables}

4.1 Parameters of the Integrate and Fire neuron model . . . . . . . . . . 41

4.2 Hebbian learning rule used in the model . . . . . . . . . . . . . . 46 



\section{Publications}

\section{Journals}

Faghihi, F., Kolodziejski, C., Fiala, A., Wörgötter F., Tetzlaff, C. (2013). An information theoretic model of information processing in the Drosophila olfactory system: the role of inhibitory neurons for systeme fficiency , Frontiers in Computational Neuroscience, $7: 183$

\section{Conferences}

1. Information processing in the Drosophila Olfactory System: From Odors to Kenyon cells Faramarz Faghihi, Florentin Wörgötter and Christoph Kolodziejski BCCN conference (2012)

2. Retrograde Signaling Based Model of Associative Learning in Drosophila Olfactory System Faramarz Faghihi1, Ahmed Moustafa, Ralf Heinrich and Florentin Wörgötter BCCN conference (2013) 



\title{
Curriculum Vitae
}

\section{Personal Details:}

\author{
Family name: Faghihi \\ First name: $\quad$ Faramarz \\ Nationality: Iranian \\ Date of birth: 08/14/1973 \\ Place of birth: Tehran, Iran
}

\section{Education:}

Bachelor of Science in Microbiology,faculty of sciences, Department of Biology, Tehran University, Iran.

Master of Science in Medical Biochemistry, faculty of Pharmaceutical Sciences Isfahan University of medical sciences, Iran.

PhD student of Theoretical and Computational Neuroscience, Göttingen University, Germany (October 2010-now)

\section{Internship:}

Graduate school of computing in medicine and life sciences, Institute for theoretical computer sciences, University of Lübeck, Lübeck, Germany ( 2008 )

Project: DNA Compression Algorithms 
List of Tables

\section{Research experience:}

1. Research assistant: Nano-biotechnology Research Centre, Avicenna Research Institute, Iran (2005-2007)

Project: Gene expression data analysis, Homology Modeling.

Supervisor: Dr. Mahmood Chamankhah

2. Research assistant: Institute of Pharmacology, University of Heidelberg, Heidelberg, Germany (2008)

Project: Sumoylation motifs prediction of genes involved in pain.

Supervisor: Prof. Dr. Rohini Kuner

3. Research assistant: Center of Medical Technology, University of Heidelberg, Germany (2009)

Project: Simulation of Heat induced Repetitive Discharge Pattern of DRG neurons in Rat.

Supervisor: Prof. Dr. Med. Rolf-Detlef Treede 


\section{Acknowledgements}

First of all, I am very grateful to my supervisor Prof. Dr. Florentin Wörgötter for giving me the opportunity to work on the project. His continuous support, his helpful advice in combination with the possibility to discuss new ideas and results, guided me through my PhD time.

Thanks to Prof. Dr. Ralf Heinrich to support my hypothesis and model and to provide experiments to test the model.

I would further like to express my gratitude to my thesis committee members Prof. Dr. André Fiala and Prof. Dr. Martin Göpfert for their interest in my project and scientific input.

Special thanks to Dr. Christoph Kolodziejski ,Dr. Christian Tetzlaff and Dr. Ahmed A.Moustafa for their helps to develop the models.

Finally, I would never be able to conclude my $\mathrm{PhD}$ without the continuous support and helps of my dear wife Sara. 